\section{Oligodeoxyribonucleotide Analogs Functionalized with Phosphonoacetate and Thiophosphonoacetate Diesters}

Phosphonoacetate and thiophosphonoacetate oligodeoxyribonucleotides are analogs wherein the phosphodiester backbone is replaced with phosphonoacetate or thiophosphonoacetate internucleotide linkages (Fig. 4.24.1). The reactive $N, N$ (diisopropylamino)phosphinyl acetate monomers are convenient to prepare from standard protected nucleosides and give high-yield synthesis of oligodeoxyribonucleotides on solid supports. Phosphonoacetate and thiophosphonoacetate oligodeoxyribonucleotides are highly stable to nucleases. They are water-soluble and anionic at neutral $\mathrm{pH}\left(\mathrm{p} K_{\mathrm{a}}\right.$ values of 3.8 and 3.9). They hybridize with complementary DNA or RNA to yield duplexes having stabilities comparable to those achieved with phosphorothioate DNA. Both analogs stimulate RNase H degradation of complementary RNA. Chimeric oligomers end-capped with phosphonoacetate or thiophosphonoacetate internucleotide linkages have been shown to be more active than natural DNA at stimulating RNase $\mathrm{H}$ in vitro.

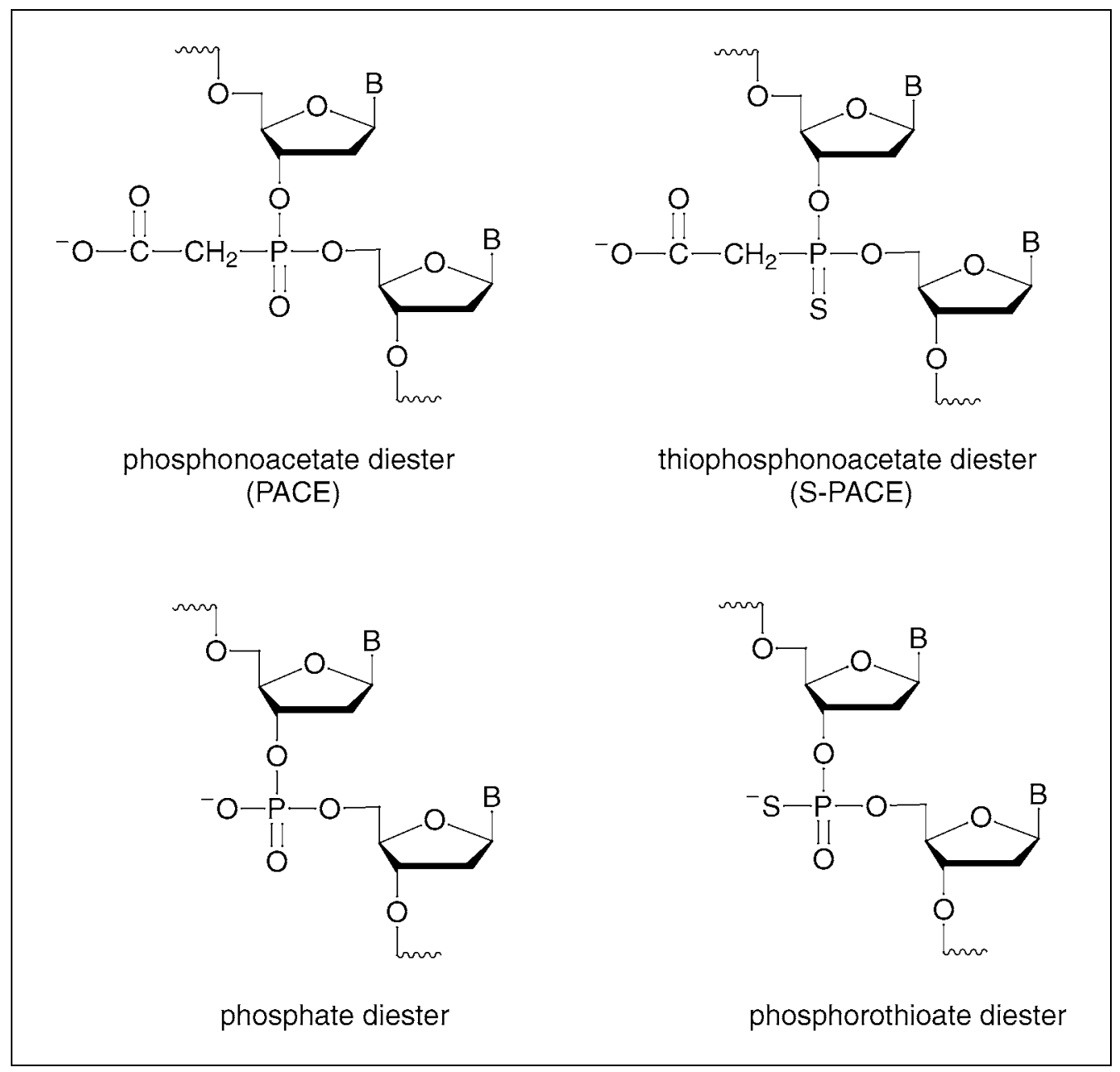

Figure 4.24.1 Structures of phosphonoacetate, thiophosphonoacetate, phosphodiester, and phosphorothioate internucleotide linkages. B: thymin-1-yl, cytosin-1-yl, adenin-9-yl, or guanin-9-yl.

Contributed by Douglas J. Dellinger, Christina M. Yamada, and Marvin H. Caruthers

Current Protocols in Nucleic Acid Chemistry (2004) 4.24.1-4.24.26

Copyright $(C) 2004$ by John Wiley \& Sons, Inc.
UNIT 4.24

Synthesis of

Modified

Oligonucleotides and Conjugates

\subsection{1}

Supplement 18 


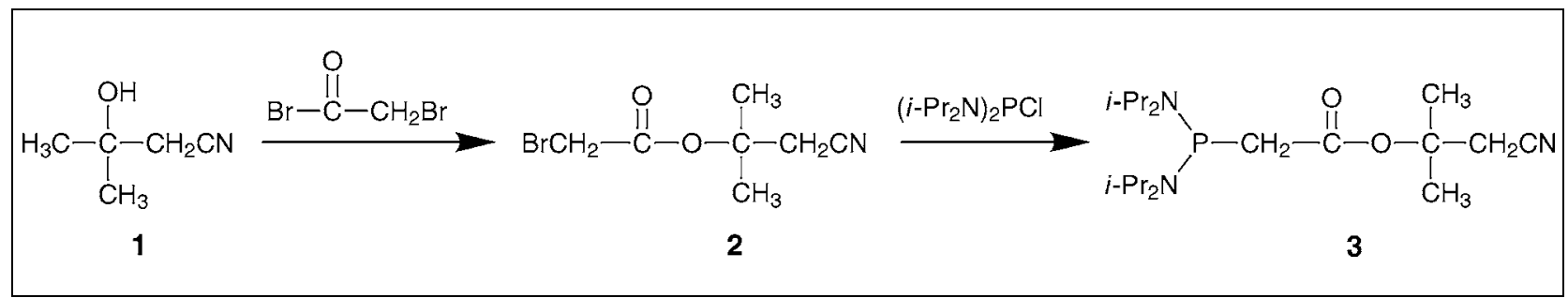

Figure 4.24.2 Synthesis of 1,1-dimethyl-2-cyanoethyl [bis( $N, N$-diisopropylamino)phosphinyl]acetate.

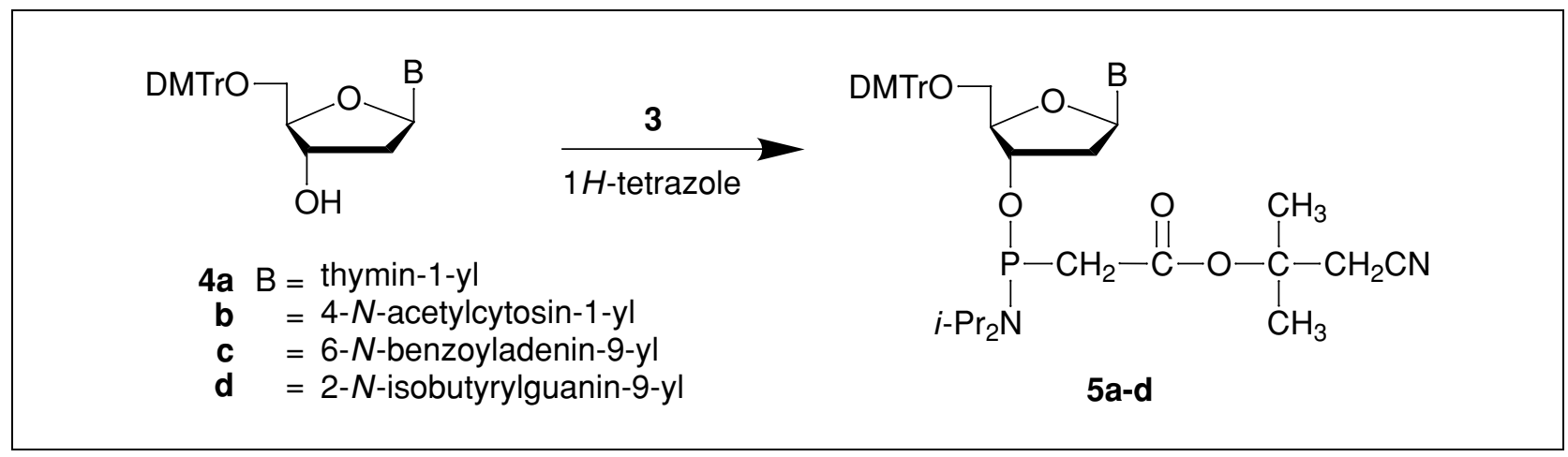

Figure 4.24.3 Preparation of protected 2'-deoxynucleoside-3'-O-(N,N-diisopropylamino)phosphinyl acetates. DMTr, 4,4'dimethoxytrityl.

\section{BASIC PROTOCOL 1}

Oligonucleotides with Phosphonoacetate and Thiophosphonoacetate Diesters

\subsection{2}

Modified oligodeoxyribonucleotides containing thiophosphonoacetate linkages with stable, intact esters are readily taken up by cells in the absence of cationic lipids.

This unit describes the solid-phase synthesis and purification of modified oligodeoxyribonucleotides (ODNs) having phosphonoacetate (PACE) and thiophosphonoacetate (S-PACE) internucleotide linkages (Dellinger et al., 2003). The first protocol outlines the preparation of appropriate synthons (Figs. 4.24.2 and 4.24.3; see Basic Protocol 1). The next two protocols illustrate the synthesis of PACE or S-PACE modified DNA (Fig. 4.24.4; see Basic Protocol 2) and chimeric oligomers (Sheehan et al., 2003) having either of these analogs combined with natural or phosphorothioate internucleotide linkages (Fig. 4.24.5; see Basic Protocol 3). The final protocols describe the drying of monomers (see Support Protocol) in addition to the purification and characterization of ODNs (see Basic Protocol 4).

CAUTION: All reactions should be carried out in a well-ventilated fume hood and contact with chemicals should be avoided. It is essential that reagents, solvents, and synthons be used under anhydrous conditions in order to maximize yield and success in preparing these oligomers. To avoid incompatibilities, a solvent-resistant Teflon head pump capable of 34 liter/min and a maximum vacuum of 1.5 torr is used for all solvent removals performed on a rotary evaporator. All intermediates and products are stored in desiccators at $-20^{\circ} \mathrm{C}$, which are allowed to warm to room temperature before they are opened.

\section{SYNTHESIS OF PROTECTED 2'-DEOXYNUCLEOSIDE- $3^{\prime}-O$-( $N, N$-DIISOPROPYLAMINO)PHOSPHINYL ACETATES}

Generating protected $2^{\prime}$-deoxynucleoside-3'-O-(N,N-diisopropylamino)phosphinyl acetates requires three steps: (1) preparation of 1,1-dimethylcyanoethyl bromoacetate (S.2; Fig. 4.24.2), (2) condensation of $\mathbf{S . 2}$ with bis(diisopropylamino)chlorophosphine to yield the phosphinylating reagent 1,1-dimethyl-2-cyanoethyl [bis $(N, N$ diisopropylamino)phosphinyl]acetate (S.3), and (3) synthesis and purification of 
Fig. 4.24.3).

The first step is the synthesis of an appropriately protected bromoacetate. The 1,1dimethyl-2-cyanoethyl ester is recommended, and is prepared from 3-hydroxy-3methylbutyronitrile and bromoacetyl bromide. However, the reaction conditions for generating this reagent are quite general. Any number of alcohols can be used with these protocols to produce the corresponding esters.

The second step is synthesis of 1,1-dimethyl-2-cyanoethyl [bis( $N, N$-diisopropylamino)phosphinyl]acetate. This phosphinylating reagent is prepared by a Reformatsky reaction (Bayless and Hauser, 1954) using the zinc bromide derivative of an ester-protected acetic acid and bis( $N, N$-diisopropylamino)chlorophosphine.

The third step, preparation of protected $2^{\prime}$-deoxynucleoside- $3^{\prime}-O$ - $(N, N$-diisopropylamino)phosphinyl acetates, can be conveniently performed starting with standard $5^{\prime}$ dimethoxytrityl-protected $2^{\prime}$-deoxynucleosides. For cost and convenience, the use of $N$ acetyl, $N$-benzoyl, and $N$-isobutyryl protection are recommended for $\mathrm{dC}, \mathrm{dA}$, and $\mathrm{dG}$, respectively. These reagents can be obtained from ChemGenes. Other fast-deprotecting exocyclic amine-blocking groups can be used as well. As described in the accompanying protocols, the use of exocyclic amine-blocking groups compatible with fast deprotection conditions (such as methylamine for $15 \mathrm{~min}$ ) is important because exposure of the oligodeoxyribonucleotide products to highly basic solutions must be limited. Prolonged exposure to strong base can result in decarboxylation of PACE/S-PACE internucleotide linkages and chain cleavage. The synthesis chemistry is compatible with most modified $2^{\prime}$-deoxynucleosides. The final protected $2^{\prime}$-deoxynucleoside- $3^{\prime}-O-(N, N-$ diisopropylamino)phosphinyl acetates are purified by silica-gel column chromatography.

CAUTION: Many phosphines are highly toxic or neuroactive agents. The phosphines described in these protocols are new reagents. Additional safety precautions should always be taken when working with novel reagents of this type, as they are uncharacterized relative to toxicity. It is extremely important to prevent inhalation or skin contact with these reagents. As in any laboratory situation, proper safety equipment should include a certified working fume hood, safety glasses, a lab coat, and reagent-impenetrable gloves. All glassware used in the synthesis or purification of these reagents should be thoroughly decontaminated before removal from the fume hood.

CAUTION: Prior to initiating synthesis, prepare the fume hood to handle large amounts of gaseous $\mathrm{HBr}$. It is important to know the local regulations regarding such hazardous material and the proper way to trap or vent this acid. When trapping $\mathrm{HBr}$, be sure to carefully calculate the mole equivalents of generated acid and ensure that the trap has enough capacity. A variety of effective procedures for working with mineral acids can be found in common laboratory manuals; the method of choice will depend upon local regulations.

\section{Materials}

Argon, dry

Toluene, anhydrous (Aldrich)

Bromoacetyl bromide (S.1, Fig. 4.24.2; Aldrich)

Nitrogen, dry (optional)

50-g bottle of 3-hydroxy-3-methylbutyronitrile (Fluka)

Bis(diisopropylamino)chlorophosphine (Digital Specialty Chemicals)

Tetrahydrofuran, anhydrous (THF; Aldrich)

Zinc metal, granular (Aldrich)

Synthesis of Modified Oligonucleotides and Conjugates

4.24.3 
Acetonitrile, anhydrous

Phosporic acid/CD ${ }_{3} \mathrm{CN}$ NMR standard

Reagent-grade hexanes, anhydrous

5'-O-(4,4'-Dimethoxytrityl)-protected 2'-deoxynucleoside (S.4; ChemGenes):

$5^{\prime}-O$-DMTr- $N^{6}$-benzoyl-2'-deoxyadenosine

$5^{\prime}-O$-DMTr- $N^{4}$-acetyl-2'-deoxycytidine

$5^{\prime}$-O-DMTr- $N^{2}$-isobutyryl-2'-deoxyguanosine

$5^{\prime}$-O $O$-DMTr-2'-deoxythymidine

Dichloromethane, anhydrous (Aldrich)

$0.45 \mathrm{M}$ tetrazole in acetonitrile (Glen Research)

Ethyl acetate, reagent grade

Diisopropylethylamine, anhydrous (DIPEA; Aldrich)

Silica gel 60 for medium-pressure liquid chromatography (MPLC; 230 to 400

mesh; Aldrich)

$120^{\circ} \mathrm{C}$ oven

500-mL and 1-liter round-bottom flasks with $24 / 40$ joints

Drying tubes containing calcium sulfate (Drierite) and equipped with a 24/40 joint

PTFE-coated stir bar

125-mL, 250-mL, and 2-L Erlenmeyer flasks

Top-loading balance

Addition funnels with 24/40 joints, pressure-equalization arms (100- and 500-mL capacity), and PTFE stopcocks

325-mm Friedrich's condensers with 24/40 joints (Labglass)

Acid vapor trap and acid-resistant tubing

Heating mantle to fit a 1-liter round-bottom flask, with controller

Rotary evaporator with solvent-resistant Teflon head pump

1- and 2-liter recovery flasks (Labglass)

Rotary evaporator trap (Labglass)

Short-path distillation apparatus (Labglass)

Three-flask distribution receiver

Vacuum pump with an inlet acid vapor trap

Capillary bleed tube (Kontes Glass) or needle valve attached to a Y-connector

Powder funnels

24/40 rubber septa

Hand-held heat gun (Aldrich)

500 -mL separatory funnel

Ground-glass 24/40 joint

50-mL glass pipet

Glass-backed silica-gel TLC plates with fluorescent indicator (Aldrich)

TLC developing tank (Aldrich)

UV viewing cabinet (Aldrich)

Flash-chromatography column (Aldrich cat) and flow controller

Sea sand

350-mL fritted glass Buchner funnel, medium porosity, fitted with a 24/40 vacuum adapter (Labglass)

Vacuum desiccator and solid PTFE vacuum pump with maximum vacuum of 1.5 torr (Aldrich)

Additional reagents and equipment for ${ }^{31} \mathrm{P}$ NMR, thin-layer chromatography (TLC; APPENDIX 3D), column chromatography (APPENDIX $3 E$ ), and fast atom bombardment mass spectrometry (FAB-MS)

Oligonucleotides 
1. Dry all glassware overnight in an oven at $120^{\circ} \mathrm{C}$.

2. Remove the 1-liter round-bottom flask with $24 / 40$ joint from the oven and cool under a stream of dry argon. Place the flask in a fume hood and secure it on a magnetic stir plate.

3. Add $500 \mathrm{~mL}$ anhydrous toluene and a PTFE-coated stir bar to the flask. Cap with a drying tube. Secure the entire apparatus to the fume hood's lattice support or a support stand.

4. Remove the 125-mL Erlenmeyer flask from the oven and cool to room temperature under a stream of dry argon. Tare the flask in the hood using a top-loading balance.

5. Weigh out $108 \mathrm{~g}(600 \mathrm{mmol})$ bromoacetyl bromide (S.1) into the flask and then quickly transfer to the toluene-containing flask by temporarily removing the drying tube, adding the bromoacetyl bromide, and then reattaching the drying tube.

6. Rinse the Erlenmeyer flask with anhydrous toluene and add the rinse to the solution. Stir on the magnetic stir plate to completely dissolve the bromoacetyl bromide.

7. Remove the $100-\mathrm{mL}$ addition funnel from the oven and cool to room temperature using a stream of dry nitrogen or argon. Make sure that the PTFE stopcock is closed and tightened to prevent leaks.

8. Transfer the entire contents of a $50-\mathrm{g}(500 \mathrm{mmol})$ bottle of 3-hydroxy-3methylbutyronitrile to the addition funnel. Rinse the reagent bottle with anhydrous toluene and add to the funnel.

9. Remove the drying tube from the 1-liter round-bottom flask and attach the addition funnel. Place the drying tube on the addition funnel, open the stopcock, and add 3-hydroxy-3-methylbutyronitrile dropwise to the stirred solution of bromoacetyl bromide.

3-Hydroxy-3-methylbutyronitrile is a tertiary alcohol that reacts very slowly with bromoacetyl bromide. This slow reactivity allows one to add the two components rapidly without cooling the flask. If another alcohol is used, especially a primary alcohol, the reaction can be highly exothermic at room temperature. When using another alcohol, it is important to first perform a test reaction on a small scale: cool the flask containing bromoacetyl bromide in an ice bath and add the alcohol dropwise to the stirred reaction mixture. This test reaction is used to determine how exothermic the reaction will become and how much cooling will be required for a larger-scale reaction. It is also important to remember that the efficiency of cooling the contents of a larger-volume flask with an external cooling bath decreases significantly as the ratio of surface area to volume decreases.

10. Remove the funnel and fit a 325-mm Friedrich's condenser with attached drying tube to the flask. Fix an acid vapor trap to the drying tube using acid-resistant tubing. Place the flask in a mantle and heat the reaction mixture to reflux $\left(\sim 110^{\circ} \mathrm{C}\right)$ overnight.

11. Cool the flask to room temperature and remove the toluene using a rotary evaporator. In order to minimize loss or contamination, transfer the solution to a 2-liter recovery flask and attach a rotary evaporator trap between the recovery flask and the evaporator.

Toluene is often considered a difficult solvent to remove on a rotary evaporator due to its boiling point and tendency to "bump."

12. Purify the product as an oil by vacuum distillation using a short-path distillation apparatus, three-flask distribution receiver, and vacuum pump with inlet acid vapor trap. Perform distillation at $\sim 0.1 \mathrm{mmHg}$ using a capillary bleed tube inserted into the distillation flask or a needle valve attached to a Y-connector on the vacuum line.

Synthesis of Modified Oligonucleotides and Conjugates

\subsection{5}

Supplement 18 
Distill the oil into two components: an initial minor fraction with a wide boiling range and the major fraction at $120^{\circ}$ to $122^{\circ} \mathrm{C}$. Store the major fraction up to 6 months at $-20^{\circ} \mathrm{C}$.

The product is black before distillation, colorless after, and yellow upon storage.

It is important to place an acid vapor trap in the vacuum line in order to trap residual $\mathrm{HBr}$, and to change the pump oil immediately following distillation, to prevent corrosion and seizure of the vacuum pump.

The needle valve and Y-connector give better control over the distillation pressure.

The constant boiling product (1,1-dimethylcyanoethyl bromoacetate; S.2) gave $97.3 \mathrm{~g}$ of clear, colorless liquid (88\% yield). ${ }^{1} \mathrm{H} \mathrm{NMR}\left(\mathrm{CDCl}_{3}\right): \delta 3.75(\mathrm{~s}, 2 \mathrm{H}), 2.86(\mathrm{~s}, 2 \mathrm{H}), 1.53$ $(\mathrm{s}, 6 \mathrm{H})$. Electron impact mass spectrometry (EI-MS) generated molecular ions of $205 \mathrm{~m} / \mathrm{e}$ and $207 \mathrm{~m} / \mathrm{e}$ with isotopic abundances of $51 \%$ and $49 \%$.

\section{Synthesize phosphinylating reagent}

13. Remove the 500-mL round-bottom flask with $24 / 40$ joint from the oven. Cool under a stream of dry argon, place in the fume hood, and fit with a drying tube.

14. With a balance placed in the hood, weigh $20 \mathrm{~g}(75 \mathrm{mmol})$ bis(diisopropylamino)chlorophosphine into a plastic weighing boat and then quickly transfer the phosphine to the cool, dry 500-mL flask by temporarily removing the drying tube from the flask and adding the phosphine via a powder funnel.

This reagent is moisture sensitive. It is therefore important to minimize its exposure to the atmosphere by working quickly.

15. Partially fill the flask with $300 \mathrm{~mL}$ anhydrous THF, add a stir bar, and place the flask on a magnetic stir plate. Cap the flask with the drying tube and secure to the fume hood's lattice support or a support stand.

16. Stir the solution vigorously for $1 \mathrm{hr}$ or until the phosphine has dissolved.

The bis(diisopropylamino)chlorophosphine is often contaminated with small amounts of diisopropylamine hydrochloride and H-phosphonate hydrolysis products. These contaminants are less soluble in THF than the desired phosphine and can form an insoluble precipitate. To ensure effective conversion to the product, it is recommended that the bis(diisopropylamino)chlorophosphine be evaluated by ${ }^{31} P \mathrm{PMR}$ prior to use. The chlorophosphine should appear as a singlet in anhydrous $\mathrm{CD}_{3} \mathrm{CN}$ with a chemical shift of 134.4 ppm. Peaks between 0 and 20 ppm represent various hydrolyzed or oxidized products. If peaks other than these appear in the spectrum, the reagent should be rejected or repurified (purity should be $>90 \%$ ). Purity of the chlorophosphine can be roughly estimated by integrating various peaks and using the results to determine the amount of impure phosphine to use in the reaction. The chlorophosphine can be synthesized and purified by recrystallization using the procedures described in Dellinger et al. (2003).

17. While the phosphine is being dissolved, remove the 1-liter three-neck round-bottom flask from the oven and cool under a stream of dry argon. Place it on a magnetic stir plate and secure it to the fume hood's lattice support or a support stand. Fit a drying tube to the center joint and rubber septa to the side joints.

18. Weigh $7.2 \mathrm{~g}(110 \mathrm{mmol})$ granular zinc metal into a plastic weigh boat. Temporarily remove the drying tube from the flask, immediately transfer the zinc to the flask using a powder funnel, and replace the drying tube.

It is important to keep the zinc metal dry and under inert atmosphere, as the presence of large amounts of zinc oxide can inhibit the initiation of the Reformatsky coupling reaction.

Oligonucleotides acetate and Thiophosphonoacetate Diesters

\subsection{6}

Supplement 18 
dry argon. Make sure that the PTFE stopcocks are closed and tightened to prevent leaks.

20. Working quickly, decant the THF/phosphine solution into one of the addition funnels while leaving any insoluble material in the flask. Cap the funnel with a rubber septum and place on one of the side joints of the three-neck flask. Slurry the residual insoluble precipitate into $50 \mathrm{~mL}$ anhydrous THF and pour onto the zinc in the three-neck roundbottom flask.

It is important that this step be performed quickly, as the phosphine solution is moisture sensitive.

21. Place the Erlenmeyer flask on the top-loading balance and tare. Carefully weigh $18.2 \mathrm{~g}$ (82.5 mmol) 1,1-dimethylcyanoethyl bromoacetate (S.2) into the dry Erlenmeyer flask. Add $150 \mathrm{~mL}$ anhydrous THF and swirl the flask to dissolve the reagent.

22. Pour this solution into the second addition funnel, cap the funnel with a rubber septum, and place it on the third neck of the flask.

23. Place the Friedrich's condenser in the center neck of the three-neck flask and insert a drying tube on top of the condenser. Connect the condenser to a water source and drain using appropriate tubing. Add a PTFE-coated magnetic stir bar to the three-neck flask.

24. Add one third of the phosphine $(100 \mathrm{~mL})$ and bromoacetate $(50 \mathrm{~mL})$ solutions to the flask and heat this mixture using a hand-held heat gun focused on the zinc, with the magnetic stir plate turned off, until boiling begins.

It is important to focus the heat from the heat gun onto the zinc metal.

25. Once the THF boils, remove the heat gun and observe the reaction mixture.

Assessment that the Reformatsky reaction has initiated is quite obvious as it will be noticeably exothermic, and the cloudy colorless mixture will become clear and slightly yellow. If the reaction does not initiate, activate the heat gun and locally heat the zinc until the THF once again begins to boil. Once the reaction has initiated, the solution will reflux without the use of a heat gun.

26. Continue the reaction at reflux by slowly adding the remainder of the phosphine and bromoacetate solutions. If the reaction becomes too vigorous, engage the magnetic stir plate, as stirring usually controls the rate. If it is still too vigorous, cool the flask using an ice bath.

27. Once addition is complete, keep the solution at reflux (which is lost as the reaction slows) for 30 min by using a heating mantle. Cool to room temperature.

28. Analyze by ${ }^{31} \mathrm{P}$ NMR for completeness by dissolving a few drops of the reaction mixture in an NMR tube containing anhydrous acetonitrile. First, lock the NMR instrument and shim the magnet on a phosphoric acid/ $\mathrm{CD}_{3} \mathrm{CN}$ standard. Then remove the standard, turn off the lock, insert the NMR tube with the crude sample into the instrument, and acquire experimental results.

${ }^{31} P$ NMR samples of these crude mixtures are typically evaluated in anhydrous, nondeuterated acetonitrile with the deuterium lock turned off due to the difficulty and expense of obtaining absolutely anhydrous deuterated solvents. Commercial "anhydrous" deuterated solvents falsely indicate that a significant amount of the reaction products are hydrolyzed. With the same anhydrous, nondeuterated acetonitrile commonly used for bulk reactions, a more accurate evaluation of hydrolysis products has been obtained.

The chlorophosphine $\left(\delta_{P}=135 \mathrm{ppm}\right)$ should be completely consumed and converted to a single product $\left(\mathrm{S.3} ; \delta_{P}=48 \mathrm{ppm}\right)$.

Synthesis of Modified Oligonucleotides and Conjugates

4.24.7 
29. Cool reaction mixture and decant into a 1-liter recovery flask, leaving behind the unreacted metal. Remove THF using a rotary evaporator to yield a yellow/brown viscous oil.

\section{Isolate phosphinylating reagent}

30. To isolate the product by trituration, add $250 \mathrm{~mL}$ anhydrous reagent-grade hexanes, place a rubber septum in the flask's $24 / 40$ joint, and shake vigorously to dissolve the phosphine. Allow the oil and hexanes to settle and decant the hexane layer into a 2-liter recovery flask. Repeat for a total of three successive extractions.

The oil will be converted to a solid.

31. Dissolve the resulting solid in $150 \mathrm{~mL}$ anhydrous acetonitrile, place in a 500-mL separatory funnel, and extract twice with $150-\mathrm{mL}$ portions of anhydrous hexanes.

32. Analyze the acetonitrile solution by ${ }^{31} \mathrm{P}$ NMR for absence of the product $\left(\delta_{P}=\right.$ $48 \mathrm{ppm}$ ) and discard.

33. Combine all hexane fractions in the 2-liter recovery flask and concentrate the product on a rotary evaporator to a slightly yellow oil. Redissolve in $300 \mathrm{~mL}$ anhydrous hexanes and place the flask in a $-20^{\circ} \mathrm{C}$ freezer overnight.

34. Decant the hexane solution into a clean, dry, 500-mL round-bottom flask and remove solvent using a rotary evaporator to give $16.4 \mathrm{~g}$ ( $88 \%$ yield) of a slightly yellow oil. Store up to 6 months at $-20^{\circ} \mathrm{C}$.

The product, 1,1-dimethyl-2-cyanoethyl [bis( $N, N$-diisopropylamino)phospinyl]acetate (S.3), can be further purified by recrystallization in anhydrous pentane. ${ }^{1} \mathrm{H} \mathrm{NMR}\left(\mathrm{CDCl}_{3}\right)$ : $\delta 3.48(\mathrm{~m}, 4 \mathrm{H}), 2.99(\mathrm{~s}, 2 \mathrm{H}), 2.79(\mathrm{~d}, \mathrm{~J}=3.12 \mathrm{~Hz}, 2 \mathrm{H}), 1.30(\mathrm{~m}, 24 \mathrm{H})$. EI-MS generated a molecular ion of 371 m/e with fragmentation loss of $\mathrm{CH}_{2} \mathrm{COOC}\left(\mathrm{CH}_{3}\right)_{2} \mathrm{CH}_{2} \mathrm{CN}$ at 231 m/e. ${ }^{31} \mathrm{P} \mathrm{NMR}\left(\mathrm{CD}_{3} \mathrm{CN}\right): \delta 48.1 \mathrm{ppm}$.

The authors typically only recrystalize with pentane to achieve good data from analysis by ${ }^{1} H N M R$.

\section{Synthesize (N,N-diisopropylamino)phosphinyl acetate monomers}

35. Remove a 500-mL round-bottom flask from the oven and cool under a stream of dry argon. Place the flask on a magnetic stir plate in the fume hood, add a stir bar, and attach a ground-glass joint capped with a drying tube. Fasten to the fume hood's lattice support or a support stand.

36. Weigh $10.0 \mathrm{~g}$ DMTr-protected 2 '-deoxynucleoside (S.4) into a plastic weighing boat. To avoid absorption of moisture, temporarily remove the drying tube from the flask, quickly transfer the nucleoside using a powder funnel, and return the drying tube to the flask. Add $250 \mathrm{~mL}$ anhydrous dichloromethane and begin stirring.

Occasionally the protected 2'-deoxynucleosides from commercial suppliers contain a significant amount of absorbed water. Any water in the solution will preferentially react with the phosphinylating agent and reduce the final yield of product. It is therefore prudent to test the protected 2'-deoxynucleoside/dichloromethane solution for the amount of water present using a Karl-Fisher titration prior to performing the phosphinylation reaction. (Once the shipment has been shown to be anhydrous, it can be assumed subsequent solutions made from the same batch will be anhydrous as long as care has been taken in handling and storage.) The authors use an Aquastar V-200 Karl-Fischer titrator(VWR ). A small amount of water in the protected 2'-deoxynucleoside can be neutralized by using a molar equivalent excess of the phosphinylating agent as an internal desiccant. Larger amounts of water require that the 2'-deoxynucleoside solution be evaporated to dryness on a rotary evaporator, redissolved in anhydrous pyridine, and evaporated to dryness again. The excess water is thus removed as a pyridine/water azeotrope. This procedure should be repeated until the water content of the pyridine solution is similar to the anhydrous solvent alone. It is subsequently important to completely remove all the pyridine, 
Table 4.24.1 Reactants Used to Prepare 2'-Deoxynucleoside-3'-O-(N,Ndiisopropylamino)phosphinyl Acetates

\begin{tabular}{llll}
\hline & $\begin{array}{l}\text { Protected } \\
2^{\prime} \text {-deoxynucleoside }\end{array}$ & $\begin{array}{l}\text { Phosphinylating } \\
\text { reagent }\end{array}$ & $\begin{array}{l}0.45 \mathrm{M} \\
\text { tetrazole }\end{array}$ \\
\hline $5^{\prime}-\mathrm{DMTr}-\mathrm{dA}^{\mathrm{Bz}}$ & $10 \mathrm{~g}$ & $6.8 \mathrm{~g}$ & $27.2 \mathrm{~mL}$ \\
$5^{\prime}-\mathrm{DMTr}-\mathrm{dC}^{\mathrm{Ac}}$ & $10 \mathrm{~g}$ & $7.8 \mathrm{~g}$ & $31.1 \mathrm{~mL}$ \\
$5^{\prime}-\mathrm{DMTr}-\mathrm{dG}^{i-\mathrm{Bu}}$ & $10 \mathrm{~g}$ & $7.0 \mathrm{~g}$ & $27.9 \mathrm{~mL}$ \\
$5^{\prime}-\mathrm{DMTr}-\mathrm{T}$ & $10 \mathrm{~g}$ & $8.2 \mathrm{~g}$ & $32.7 \mathrm{~mL}$ \\
\hline
\end{tabular}

as it will act as a base and neutralize the tetrazole acid catalyst. This is accomplished by using a similar azeotropic evaporation process with toluene. Once the protected 2'deoxynucleoside is free of pyridine, it is redissolved in anhydrous dichloromethane and the synthesis continued.

37. Place a top-loading balance in the hood and weigh the appropriate amount of phosphinylating reagent (S.3) into a plastic weighing boat (Table 4.24.1). Temporarily remove the drying tube, quickly transfer the phosphine to the 500-mL flask using a powder funnel, and return the drying tube to the flask. Continue stirring to dissolve completely ( $\sim 10$ to $30 \mathrm{~min})$.

The phosphine is moisture sensitive, so it is important to minimize its exposure to the atmosphere.

38. Add the volume of $0.45 \mathrm{M}$ tetrazole in anhydrous acetonitrile shown in Table 4.24.1 using a dry $50-\mathrm{mL}$ glass pipet. Stir $24 \mathrm{hr}$ at room temperature.

The volume given provides 0.8 molar equivalents tetrazole to the reaction.

39. Spot the reaction mixture on a glass-backed silica-gel TLC plate with fluorescent indicator, alongside a dichloromethane solution of the appropriate starting material. Air dry the spots.

40. Carefully place the TLC plate in a developing tank pre-equilibrated with reagentgrade ethyl acetate and allow the solvent elution front to reach the top of the plate. Air dry the plate, place in a UV viewing cabinet, and illuminate using shortwave irradiation.

The reaction is determined complete by spot-to-spot conversion to a faster eluting product. For additional details on TLC, see APPENDIX $3 D$.

41. Confirm conversion to product by loss of the ${ }^{31} \mathrm{P}$ NMR signal corresponding to $\mathbf{S . 3}$ $(\delta=48.1 \mathrm{ppm})$.

42. If conversion is incomplete by these assays, allow the reaction mixture to stir another $24 \mathrm{hr}$.

43. Quench the reaction by adding 0.8 molar equiv anhydrous DIPEA and stirring 5 min.

44. Pour the solution into a 1-liter recovery flask and concentrate to a viscous oil using a rotary evaporator to remove solvent. Proceed to purification immediately.

\section{Purify product by column chromatography}

45. For each preparation, place $\sim 700 \mathrm{~mL}$ dry silica gel 60 (230 to $400 \mathrm{mesh}$ ) and $700 \mathrm{~mL}$ solvent (Table 4.24.2) in a 2-liter Erlenmeyer flask and swirl to equilibrate. If needed, use additional solvent to assure that the silica gel is completely solvated and easy to pour. Pour $\sim 16 \mathrm{~cm}$ solvated silica gel into a flash chromatography column and use solvent to wash any silica from the sides of the column.

For additional details on column chromatography, see APPENDIX $3 E$.

Synthesis of Modified Oligonucleotides and Conjugates

4.24.9 
Table 4.24.2 Column Elution Solvents for Various Synthons

\begin{tabular}{lll}
\hline $2^{\prime}$-Deoxynucleoside base & $\%$ Ethyl acetate $(\mathrm{v} / \mathrm{v})$ & $\%$ Hexanes $(\mathrm{v} / \mathrm{v})$ \\
\hline Adenine & 100 & 0 \\
Cytosine & 50 & 50 \\
Guanine & 100 & 0 \\
Thymine & 100 & 0 \\
\hline
\end{tabular}

46. Assemble the column in the fume hood by tightly clamping it to the fume hood's lattice support or a support stand. Attach the flow controller. Push solvent through the column at a flow rate of $\sim 300 \mathrm{~mL} / \mathrm{min}$ using air or nitrogen to compress the silica gel bed. Remove the pressure and flow controller when the solvent level reaches the compressed silica. Sprinkle sea sand on the silica gel bed to a depth of $\sim 2 \mathrm{~cm}$.

47. Carefully pour an additional $500 \mathrm{~mL}$ solvent into the column without disturbing the compressed silica gel bed. Reattach the flow controller and push the liquid through the column at the same rate until it reaches the silica gel bed.

Because most silica gels are acidic, it is typical to add a small percentage of triethylamine to the eluting solvent when purifying protected 2 '-deoxynucleoside phosphoramidites. This prevents loss of the DMTr protecting group and acid-catalyzed hydrolysis of the phosphoramidite. However, the presence of triethylamine in the eluting solvent has a deleterious effect on the stability of the $2^{\prime}$-deoxynucleoside-3'-O-(N,N-diisopropylamino)phosphinyl acetates. As a result, losses in recovered yield are greater in the presence of triethylamine than when this base is not used. Therefore, addition of triethylamine to these solvents is not recommended. Typically, there is a considerable amount of diisopropylammonium tetrazolide in the crude $2^{\prime}$-deoxynucleoside-3'-O-(N,N-diisopropylamino)phosphinyl acetate products to help neutralize excess acid on the silica gel. However, detritylation has still been observed infrequently during column chromatography. Detritylation on the column is easily detectable as a bright orange band on the silica column. To eliminate this detritylation, a small amount of TEA may be used to neutralize excess acid on silica gel during column packing and equilibration, but excess amine should be thoroughly flushed from the column prior to adding the crude material to the top of the column. Under either of these conditions, it is important to run the column at a rapid flow rate and not leave the product in contact with the silica gel for prolonged periods of time. The DMTrprotected $2^{\prime}$-deoxythymidine-3'-O-(N,N-diisopropylamino $)$ phosphinyl acetates seem to be most susceptible to degradation during prolonged exposure to silica gel. When purifying these synthons, it is important to elute the column rapidly but carefully and not pause during purification.

48. Dissolve the crude product (step 43) in a minimum volume of ethyl acetate and add to the column by slowly dropping the mixture onto the sand layer using a Pasteur pipet. Use a small volume of solvent $(50 \mathrm{~mL})$ to wash the flask and add this solution dropwise to the sand layer.

49. Push the reaction mixture and solvent onto the silica gel by briefly pressurizing the column until the solvent level once again reaches the top of the compressed bed. Carefully add an additional $50 \mathrm{~mL}$ solvent to the sand layer and once again push it onto the silica gel by briefly pressurizing the column until the liquid level has reached the compressed bed.

Oligonucleotides with Phosphonoacetate and Thiophosphonoacetate Diesters 4.24.10 
in the UV viewing cabinet using shortwave irradiation. Keep samples containing UV-absorbing material and discard those without.

52. For detailed analysis, spot TLC plates with column fractions that contain UV-absorbing material along with a dichloromethane solution of the appropriate starting material and the crude reaction mixture. Air dry the spots, place the plate in a developing tank pre-equilibrated with ethyl acetate, and elute until the solvent front reaches the top of the plate. Air dry and view in the UV viewing cabinet using shortwave irradiation.

53. Identify column fractions containing pure material suspected of being the product. Combine in a recovery flask and remove solvent on a rotary evaporator.

The resulting products are usually glassy or foamed solids. The authors frequently elect to convert them to granular powders by precipitation into anhydrous hexanes (step 54), because powders are easier to manipulate for solid-phase DNA synthesis.

\section{Work up final product}

54. For each preparation, place 1 liter anhydrous hexanes in a 2-liter recovery flask. Redissolve the purified solid in a minimal amount of anhydrous dichloromethane and add dropwise to the hexanes using a Pasteur pipet. Add the DCM/product mixture a few drops at a time, rapidly swirling the solution.

A white solid, corresponding to the product, precipitates from the hexanes.

55. Once addition is complete, isolate the solid by filtration using a $350-\mathrm{mL}$ mediumporosity, fritted glass Buchner funnel fitted with a 24/40 vacuum adapter. During filtration, keep the solid submersed in solvent to prevent the granular precipitate from converting to a gum.

56. When filtration is complete, immediately place the funnel in a vacuum desiccator and attach to a two-stage vacuum pump or high-vacuum line. Make sure that the vacuum line or vacuum pump is "trapped" so that there is no chance that oil will back up into the vacuum desiccator. Allow the precipitate to be evacuated in the vacuum desiccator for at least $12 \mathrm{hr}$ in order to remove all residual solvent.

Rapid evacuation of solvent from the powder can often lead to an "explosion" of the product such that it covers the inside walls of the desiccator. This can be prevented by slowly evacuating the desiccator in stages. Additionally, covering the funnel with aluminum foil containing a few small holes can help prevent this unfortunate loss of product.

57. Once the precipitate has been dried under vacuum, close the stopcock to isolate the vacuum desiccator, remove the high-vacuum line, and bleed the desiccator slowly with dry argon or nitrogen.

The resulting white powder is easily partitioned into DNA synthesizer reagent vials.

58. Identify and analyze each product for purity by ${ }^{31} \mathrm{P}$ NMR and FAB-MS. Store up to 6 months at $-20^{\circ} \mathrm{C}$.

1,1-Dimethyl-2-cyanoethyl $\quad 5^{\prime}$-O-(4,4'-dimethoxytrityl)-2'-deoxythymidine-3'-O-(N,Ndiisopropylamino)phosphinyl acetate (S.5a) is isolated with an average yield of $84 \%$.

${ }^{31} \mathrm{P} \mathrm{NMR}\left(\mathrm{CD}_{3} \mathrm{CN}\right): \delta$ 120.3, $120.8 \mathrm{ppm}$. FAB-HRMS: calcd. for $\mathrm{C}_{44} \mathrm{H}_{55} \mathrm{~N}_{4} \mathrm{O}_{9} \mathrm{P}(\mathrm{M}+\mathrm{H})^{+}$ 815.3785, found 815.3775 .

1,1-Dimethyl-2-cyanoethyl 5'-O-(4,4'-dimethoxytrityl)- $N^{4}$-acetyl-2'-deoxycytidine-3'-O$(\mathrm{N}, \mathrm{N}$-diisopropylamino)phosphinyl acetate $(\boldsymbol{S} .5 \boldsymbol{b})$ is isolated with an average yield of 78\%. ${ }^{31} \mathrm{P} \mathrm{NMR}\left(\mathrm{CD}_{3} \mathrm{CN}\right): \delta$ 121.3, $121.8 \mathrm{ppm}$. FAB-HRMS: calcd. for $\mathrm{C}_{45} \mathrm{H}_{56} \mathrm{~N}_{5} \mathrm{O}_{9} \mathrm{P}$ $(M+H)^{+} 842.3894$, found 842.3914 .

1,1-Dimethyl-2-cyanoethyl $\quad 5^{\prime}$-O-(4,4'-dimethoxytrityl)- $N^{6}$-benzoyl-2'-deoxyadenosine$3^{\prime}-O-(N, N$-diisopropylamino)phosphinyl acetate $(\mathbf{S . 5 c})$ is isolated with an average yield

Synthesis of Modified Oligonucleotides and Conjugates

\subsubsection{1}

Supplement 18 
of 75\%. ${ }^{31} \mathrm{P} \mathrm{NMR}\left(\mathrm{CD}_{3} \mathrm{CN}\right): \delta$ 120.8, 121.6 ppm. FAB-HRMS: calcd. for $\mathrm{C}_{51} \mathrm{H}_{58} \mathrm{~N}_{7} \mathrm{O}_{8} \mathrm{P}$ $(M+H)^{+} 928.4163$, found 928.4159 .

1,1-Dimethyl-2-cyanoethyl 5'-O-(4,4'-dimethoxytrityl)- $N^{2}$-isobutyryl-2'-deoxyguanosine$3^{\prime}-O-(N, N$-diisopropylamino)phosphinyl acetate $(\boldsymbol{S . 5 d})$ is isolated with an average yield of 89\%. ${ }^{31} \mathrm{P} N M R\left(C D_{3} C N\right): \delta$ 121.7, 122.0 ppm. FAB-HRMS: calcd. for $\mathrm{C}_{48} \mathrm{H}_{60} \mathrm{~N}_{7} \mathrm{O}_{9} \mathrm{P}-$ $(M+H)^{+}$910.4268, found 910.4235 .

BASIC

PROTOCOL 2

\section{AUTOMATED SYNTHESIS OF PHOSPHONOACETATE AND THIOPHOSPHONOACETATE DNA}

\section{Phosphonoacetate DNA Synthesis}

The chemical synthesis of PACE ODNs (Fig. 4.24.4) can be accomplished manually, but the use of an automated DNA synthesizer is preferred. This protocol describes the synthesis as performed on an ABI model 394 automated DNA synthesizer. The chemistry is completed on a solid support with standard controlled-pore glass (CPG) DNA synthesis columns with long-chain alkylamino (LCAA) linker ( $1 \mu \mathrm{mol} \mathrm{dA}, \mathrm{Ac}-\mathrm{dC}, \mathrm{dG}, \mathrm{dT} ; 500-\AA$ glass pore size; Glen Research). When using a dC-containing CPG column, it is important that the $\mathrm{dC}$ be protected with an acetyl rather than benzoyl group. The use of the acetyl protecting group on $\mathrm{dC}$ allows for the rapid deprotection of the ODNs using methylamine at $55^{\circ} \mathrm{C}$ for $15 \mathrm{~min}$ without methylation of the $\mathrm{N} 4$ position of the cytosine residues. The use of the more typical benzoyl-protected $\mathrm{dC}$ monomers and CPG columns results in a small but detectable amount of methylation of the N4 position during ODN deprotection using these rapid conditions. The use of the typical benzoyl-protected dA and isobutyrylprotected $\mathrm{dG}$ is compatible with rapid ODN deprotection using methylamine and does not result in detectable methylation of these heterobases.

The recommended synthesis cycle is adapted from a standard 1- $\mu$ mol 2-cyanoethylphosphoramidite DNA cycle (Caruthers et al., 1987). The coupling wait-time is increased to $1998 \mathrm{sec}$ by using two successive 999 -sec wait steps. The oxidation step is accomplished prior to the capping step; the oxidation wait time is $180 \mathrm{sec}$. Table 4.24 .3 summarizes the synthesis cycle as applied to the 394 automated synthesizer. A general description of automated DNA synthesis can be found in APPENDIX $3 C$.

The monomers used for PACE ODN synthesis are the 1,1-dimethyl-2-cyanoethyl 5'$O$-(4,4'-dimethoxytrityl)-2'-deoxynucleoside-3'-O-( $N, N$-diisopropylamino)phosphinyl acetates described above (see Basic Protocol 1). They are also commercially available from MetaSense Technologies (see http://www.Metasensetechnologies.com for ordering and helpful information). It is important to dry the protected $2^{\prime}$-deoxynucleoside$3^{\prime}-O$-( $N, N$-diisopropylamino)phosphinyl acetates overnight to remove any possible moisture before use (see Support Protocol). Indeed, prior to dissolving the synthons, researchers should familiarize themselves with techniques used for handling air-sensitive or moisture-sensitive reagents. The most common failure modes for DNA synthesis result from moisture contamination of amidites or activators. A good treatise on this subject, Aldrich Technical Bulletin: AL-134 Handling AirSensitive Reagents, can be obtained from Aldrich Chemical in PDF format at http://www.sigmaaldrich.com/aldrich/bulletin/al_techbull_al134.pdf.

Freshly sublimed tetrazole dissolved in anhydrous acetonitrile at a concentration of $0.45 \mathrm{M}$ is used as an activator. A prepared solution of $0.45 \mathrm{M}$ tetrazole in acetonitrile can be purchased from Glen Research. Trichloroacetic acid (3\% w/v in anhydrous dichloromethane; Glen Research) is used to deprotect the 5'-DMTr group prior to each round of coupling. Capping is accomplished using a two-part capping solution modified from Hogrefe et al. (1993). Cap A consists of 10\% acetic anhydride in anhydrous tetrahydrofuran (Glen Research), and Cap B consists of $0.625 \%$ (w/v)

\subsubsection{2}




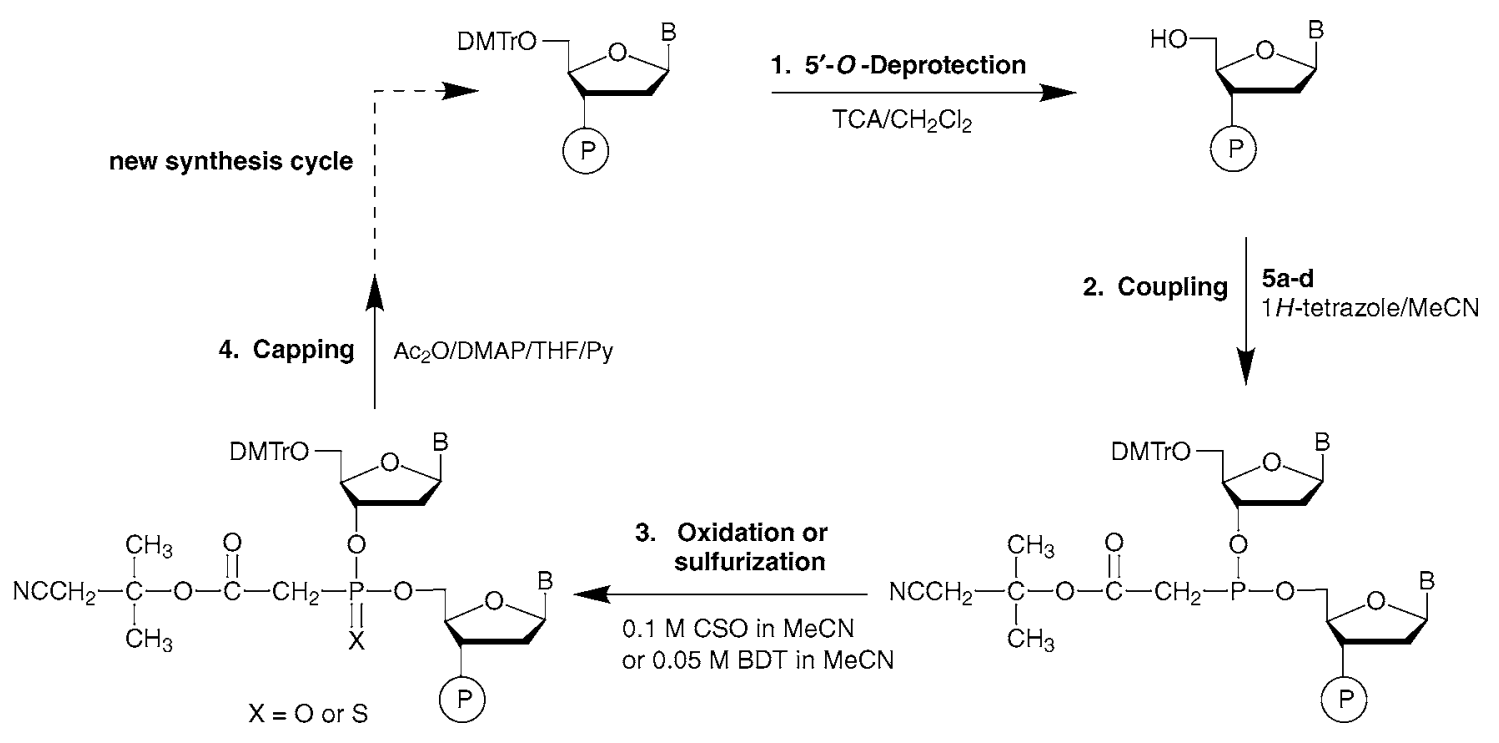

Figure 4.24.4 Synthesis scheme for preparing phosphonoacetate and thiophosphonoacetate oligodeoxyribonucleotides. Circled $\mathrm{P}$ is the LCAA-CPG support (long-chain alkylamino controlled-pore glass hemisuccinate). Abbreviations: $\mathrm{B}$, thymin1-yl, 4-N-acetylcytosin-1-yl, 6-N-benzoyladenin-9-yl, or 2- $N$-isobutyrylguanin-9-yl; BDT, 3H-1,2-benzodithiol-3-one 1,1dioxide; CSO, (1S)-(+)-(10-camphorsulfonyl)oxazaridine; DMAP, 4-dimethylaminopyridine; DMTr, 4,4'-dimethoxytrityl; Py, pyridine; TCA, trichloroacetic acid.

Table 4.24.3 Synthesis Cycle for Phosphonoacetate or Thiophosphonoacetate DNA

\begin{tabular}{|c|c|c|c|}
\hline Step & Delivery time (sec) & Wait time (sec) & Port \\
\hline 1. Dichloromethane & 35 & - & 19 \\
\hline 2. Detritylation & 85 & - & 14 \\
\hline 3. Acetonitrile & 20 & - & 18 \\
\hline 4. Coupling ${ }^{a}$ & $2.5 \times 2$ & $999 \times 2$ & $5-8,9$ \\
\hline 5. Acetonitrile & 10 & - & 18 \\
\hline 6. Oxidation or & 8 & 180 & 15 \\
\hline sulfurization $^{b}$ & 8 & 60 & 10 \\
\hline 7. Acetonitrile & 40 & - & 18 \\
\hline 8. Capping & 10 & 10 & 11 and 12 \\
\hline 9. Acetonitrile & 30 & - & 18 \\
\hline
\end{tabular}

${ }^{a}$ Coupling involves simultaneous delivery of an appropriately protected $2^{\prime}$-deoxynucleoside- $3^{\prime}-O-(N, N$ diisopropylamino)phosphinyl acetate (ports 5 to 8 ) and tetrazole (port 9).

${ }^{b}$ Synthesis of phosphonoacetate DNA requires placement of CSO at port 15 , whereas synthesis of thiophosphonoacetate DNA requires BDT at port 10. The appropriate wait time is then programmed into the cycle.

4- $N, N$-dimethylaminopyridine (DMAP, Aldrich) in anhydrous pyridine (Aldrich). Oxidation of the nascent internucleotide phosphonite to the phosphonate is carried out with $0.1 \mathrm{M}(1 S)-(+)(10$-camphorsulfonyl)oxaziridine (Aldrich) in anhydrous acetonitrile $(1 \mathrm{~g}$ $\mathrm{CSO}$ in $43.6 \mathrm{~mL} \mathrm{CH}{ }_{3} \mathrm{CN}$ ). The acetonitrile used should be anhydrous synthesis grade, and dichloromethane should be certified ACS grade (both available from Fisher Biotech).

\section{Thiophosphonoacetate DNA Synthesis}

With the exception of the sulfurization step, the synthesis of S-PACE ODNs is analogous to the procedure used for PACE DNA. Because the same synthons are used to prepare both

Synthesis of Modified

Oligonucleotides and Conjugates

4.24.13

Supplement 18 
SUPPORT PROTOCOL
PACE and S-PACE ODNs, the synthesis cycle in Table 4.24.3 can be followed, except that $3 \mathrm{H}$-1,2-benzodithiol-3-one-1,1-dioxide (BDT) is used as a sulfurization reagent in place of the CSO used for oxidation in PACE DNA synthesis. The sulfurization wait time is $60 \mathrm{sec}$, and the sulfurization solution is $0.05 \mathrm{M}$ BDT (Glen Research) in anhydrous acetonitrile ( $1 \mathrm{~g}$ BDT in $\left.100 \mathrm{~mL} \mathrm{CH}_{3} \mathrm{CN}\right)$.

\section{DRYING OF PHOSPHONOACETATE AND THIOPHOSPHONOACETATE MONOMERS}

Steps are provided to describe the drying and preparation of the starting monomers prior to ODN synthesis.

\section{Materials}

1,1-Dimethyl-2-cyanoethyl 5'-O-(4,4'-dimethoxytrityl)-2'-deoxynucleoside-3'-O( $N, N$-diisopropylamino)phosphinyl acetates (S.5a-d; see Basic Protocol 1; also available from MetaSense Technologies)

Argon or nitrogen, anhydrous

Anhydrous acetonitrile, synthesis grade (Fisher Biotech)

Amber serum vials with rubber septa

$18-\mathrm{G} \times 1$-in. needle

Vacuum desiccator

Two-stage vacuum pump or high vacuum line, with trap Syringe, dry

1. Weigh the appropriate amount of each synthon into separate amber serum vials and place a rubber septum on each. Press an $18-\mathrm{G} \times 1$-in. needle through each septum until it extends about half-way. Make sure that the needles do not contact any powder.

2. Place the vials in a vacuum desiccator attached to a two-stage vacuum pump or a high vacuum line, with a trap, and evacuate for at least $12 \mathrm{hr}$ prior to use.

3. Close the stopcock to isolate the vacuum desiccator, remove the high vacuum line, and bleed the desiccator slowly with dry argon or nitrogen. Open the desiccator and quickly remove the needles from the rubber septa.

4. Dissolve dried synthons in synthesis-grade anhydrous acetonitrile at a concentration of $0.1 \mathrm{M}$ by filling a dry syringe with the required amount of solvent (Table 4.24.4) and then transferring the solvent to the amber serum vial under dry nitrogen or argon. Store up to 6 months at $-20^{\circ} \mathrm{C}$.

For the synthesis of an average 20-mer having five of each base ( $d C, d G, d T, d A)$ and on

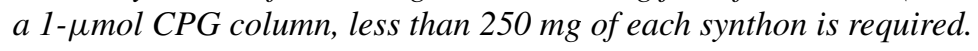

Table 4.24.4 Acetonitrile Solvent Requirements for 2'-Deoxynucleoside-3'-O(N,N-diisopropylamino)phosphinyl Acetates

Volume acetonitrile

\begin{tabular}{lll}
$2^{\prime}$-Deoxynucleoside base & $250 \mathrm{mg}$ & $1 \mathrm{~g}$ \\
\hline Adenine & $2.7 \mathrm{~mL}$ & $11.0 \mathrm{~mL}$ \\
Cytosine & $3.0 \mathrm{~mL}$ & $11.9 \mathrm{~mL}$ \\
Guanine & $2.8 \mathrm{~mL}$ & $11.0 \mathrm{~mL}$ \\
Thymine & $3.1 \mathrm{~mL}$ & $12.3 \mathrm{~mL}$ \\
\hline
\end{tabular}

Oligonucleotides with Phosphonoacetate and Thiophosphonoacetate Diesters
4.24.14

Supplement 18 
The protocol outlined in this section can be used to synthesize modified DNA having either

PACE or S-PACE internucleotide linkages combined with phosphate and thiophosphate backbones (Fig. 4.24.5). Many oligomers having these four linkages in various combinations have been synthesized, including those presented in Table 4.24.5 (also see BioSpring $\mathrm{GmbH}$, http://www.biospring.de).

\section{Preparation for Synthesis of DNA Chimeras}

The reagents and preparations for synthesis of chimeras having either PACE or S-PACE internucleotide linkages in combination with phosphate or thiophosphate backbones are analogous to those outlined in Basic Protocol 2. In order to generate S-PACE and thiophosphate linkages, the sulfurization solution used is BDT in anhydrous acetonitrile. The concentration of BDT for sulfurization remains the same for synthesizing thiophosphate and S-PACE internucleotide linkages. Oxidation to PACE and phosphate backbones is carried out with CSO; however, the concentration of CSO required to oxidize a phosphite triester to a phosphotriester (producing a normal phosphate backbone) is significantly greater than the concentration required to oxidize the phosphonite diester to the phosphonate diester (producing the PACE backbone). Effective oxidization of phosphite triesters to phosphotriesters requires a $0.5 \mathrm{M}$ solution of CSO and exposure for at least $3 \mathrm{~min}$. This higher concentration of CSO is used to oxidize both phosphite and phosphonite internucleotide linkages during the preparation of chimeras. The use of CSO for the nonaqueous oxidation of phosphite triesters is described in a Glen Report (Glen Research, 1996), which can be downloaded as a PDF file from the Glen Research Web site (http://www.glenres.com).

Synthons used to prepare PACE and S-PACE linkages (see Basic Protocol 1 or MetaSense Technologies) are dried as described in the Support Protocol, dissolved in anhydrous acetonitrile (see Table 4.24.4 for appropriate concentrations), and then transferred to ports 5 to 8 on the automated DNA synthesizer. For preparing phosphate and thiophosphate internucleotide linkages, standard base-protected synthons, $5^{\prime}-O$-(4,4'-dimethoxytrityl)-3'-O-[(N,N-diisopropylamino)(2-cyanoethoxy)phosphinyl]$2^{\prime}$-deoxyribonucleoside monomers, are used according to published procedures (Caruthers et al., 1987). They are available from Glen Research (dT-CE, Ac-dC-CE, dG-CE, and dA-CE phosphoramidites). These synthons are dissolved as $0.1 \mathrm{M}$ solutions in anhydrous acetonitrile and transferred to ports 1 to 4 on the DNA synthesizer. The capping solution is described in Basic Protocol 2.

Table 4.24.5 MALDI-TOF Mass Spectrometry of ODNs ${ }^{a}$

\begin{tabular}{llll}
\hline Abbreviation & Structure & $\begin{array}{l}\text { Calculated } \\
\text { mass }\end{array}$ & $\begin{array}{l}\text { Observed } \\
\text { mass }\end{array}$ \\
\hline PACE & $\mathrm{d}(\mathrm{CaTaCaAaAaGaTaGaGaGaCaTaGaGaTaGaAaC})$ & 6270.2 & 6271.3 \\
S-PACE & $\mathrm{d}(\mathrm{CsT}$-PsAsAsGsTsGsGsGsCsTsGsGsTsGsAsC) & 6542.1 & 6540.5 \\
PACE EO & $\mathrm{d}(\mathrm{CaTCaAAaGTaGGaGCaTGaGTaGAaC)}$ & 5933.8 & 5933.1 \\
S-PACE EO & $\mathrm{d}(\mathrm{CsT}-\mathrm{Cs}$ A-AsG-TsG-GsG-CsT-GsG-TsG-AsC) & 6205.8 & 6208.2 \\
PACE Cap & $\mathrm{d}($ CaTaCaAaAaGaT-G-G-G-C-TaGaGaTaGaAaC) & 6139.9 & 6137.2 \\
S-PACE Cap & $\mathrm{d}($ CsTsCsAsA-G-T-G-G-G-C-T-G-GsTsGsAsC) & 6163.8 & 6165.5 \\
\hline
\end{tabular}

${ }^{a}$ Nomenclature: a, phosphonoacetate; s, thiophosphonoacetate; -, phosphorothioate; the absence of a symbol between nucleoside letters corresponds to a normal phosphate linkage (e.g., CT).

Synthesis of Modified Oligonucleotides and Conjugates

4.24.15

Supplement 18 


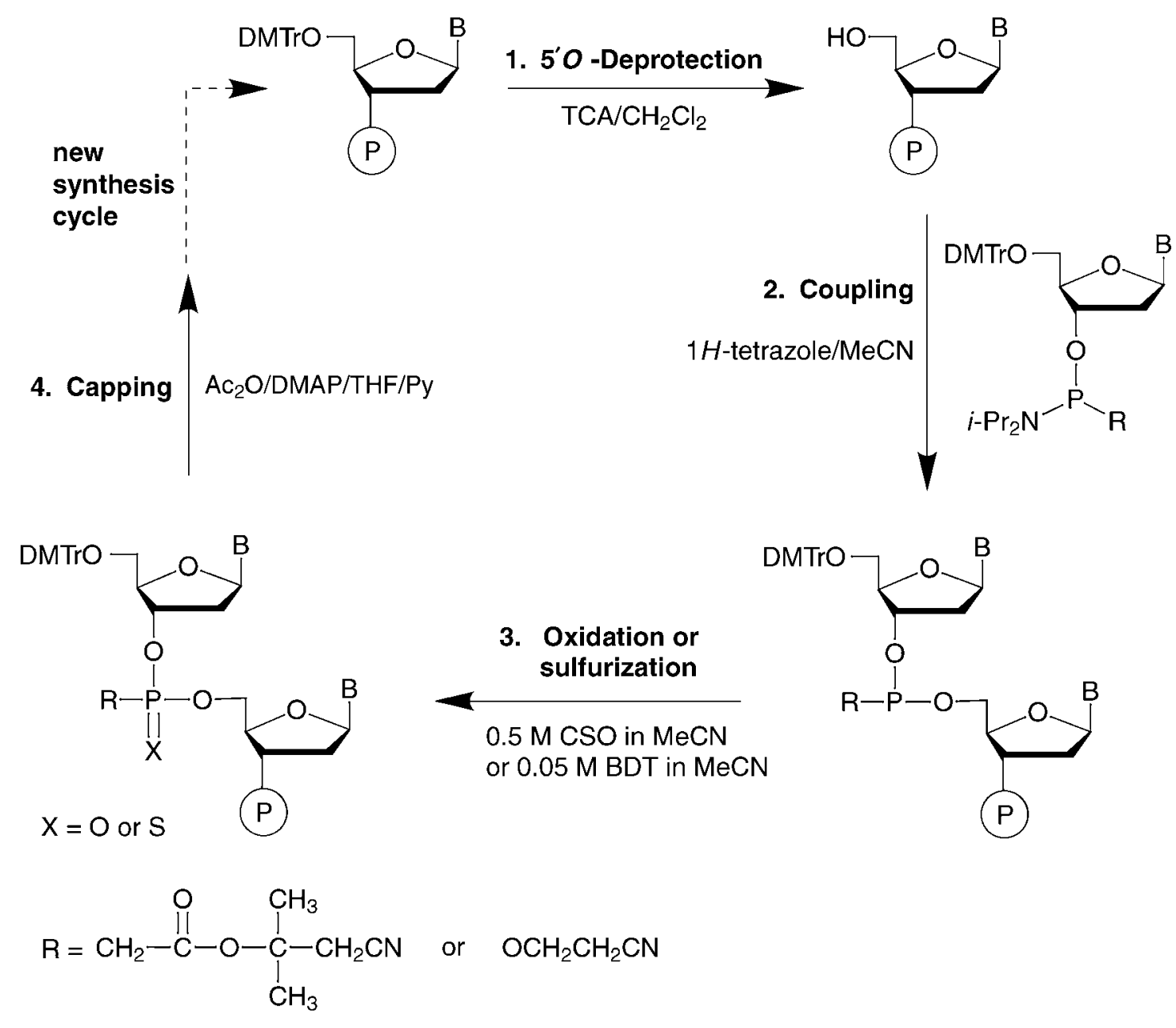

Figure 4.24.5 Synthesis scheme for preparing oligodeoxyribonucleotide chimeras having phosphonoacetate or thiophosphonoacetate internucleotide linkages combined with phosphodiester or phosphorothioate backbones. See Figure 4.24.4 for abbreviations.

Oligonucleotides with Phosphonoacetate and Thiophosphonoacetate Diesters

\section{Synthesis of Oligodeoxyribonucleotide Chimeras}

A general synthesis plan for various chimeras is outlined in Table 4.24.6. This table includes two series of synthons-i.e., the standard $2^{\prime}$-deoxynucleoside- $3^{\prime}$ phosphoramidites and the $2^{\prime}$-deoxynucleoside- $3^{\prime}-O$ - $(N, N$-diisopropylamino)phosphinyl acetates - and two choices for the $\mathrm{P}(\mathrm{III})$ to $\mathrm{P}(\mathrm{V})$ oxidation of the internucleotide linkage-i.e., CSO and BDT. Each cycle, which outlines the steps for synthesizing one internucleotide linkage, uses only one synthon and oxidation condition. In some cases, chimeras having all four bases can be synthesized using one cycle. For example, phosphodiester/PACE or phosphorothioate/S-PACE chimeras are prepared using only one oxidant (CSO or BDT, respectively). Chimeric syntheses of phosphorothioate/PACE or phosphodiester/S-PACE ODNs require two separate cycles: one for the phosphorothioate and S-PACE linkages (using the sulfurizing reagent BDT) and another to generate phosphodiester and PACE internucleotide backbones (using the oxidant CSO).

A chimera requiring two cycles is illustrated by the PACE Cap ODN shown in Table 4.24.5. This oligomer has phosphorothioate linkages flanked by PACE at the $3^{\prime}$ and $5^{\prime}$ ends. In this case, a heptamer having a PACE backbone is first synthesized using appropriate $2^{\prime}$-deoxynucleoside- $3^{\prime}-O-(N, N$-diisopropylamino)phosphinyl acetates as synthons and CSO as oxidant. At this point, BDT as a sulfurizing agent is substituted for CSO. This

\subsubsection{6}


Table 4.24.6 Synthesis Cycle for ODN Chimeras

\begin{tabular}{|c|c|c|c|}
\hline Step & Delivery time (sec) & Wait time (sec) & Port \\
\hline 1. Dichloromethane & 35 & - & 19 \\
\hline 2. Detritylation & 85 & - & 14 \\
\hline 3. Acetonitrile & 20 & - & 18 \\
\hline \multicolumn{4}{|l|}{ 4. Coupling ${ }^{a}$ : } \\
\hline standard phosphoramidites & $2.5 \times 2$ & 25 & $1-4,9$ \\
\hline phosphinoamidites & $2.5 \times 2$ & $999 \times 2$ & $5-8,9$ \\
\hline 5. Acetonitrile & 10 & - & 18 \\
\hline 6. Oxidation $o r$ & 8 & 180 & 15 \\
\hline sulfurization $^{b}$ & 8 & 60 & 10 \\
\hline 7. Acetonitrile & 40 & - & 18 \\
\hline 8. Capping & 10 & 10 & 11 and 12 \\
\hline 9. Acetonitrile & 30 & - & 18 \\
\hline
\end{tabular}

${ }^{a}$ For each cycle, coupling involves simultaneous delivery of a standard protected $2^{\prime}$-deoxynucleoside- $3^{\prime}$ phosphoramidite (ports 1 to 4 ) and tetrazole (port 9) or an appropriately protected $2^{\prime}$-deoxynucleoside- $3^{\prime}-O$ - $(N, N$ diisopropylamino)phosphinyl acetate (ports 5 to 8 ) and tetrazole (port 9).

${ }^{b}$ The choice of oxidation (CSO) or sulfurization (BDT) depends upon the synthesis plan.

is accomplished by reprogramming the cycle to use port 10 containing the BDT reagent in place of CSO at port 15 . Synthesis then continues using appropriately protected standard $2^{\prime}$-deoxynucleoside-3'-phosphoramidites in order to prepare a 12-mer having five phosphorothioate and six PACE linkages. Once again the cycle is changed so that CSO (port 15) replaces BDT (port 10) in the synthesis program. The final PACE internucleotide linkages are added using $2^{\prime}$-deoxynucleoside- $3^{\prime}$ - $O$-( $N, N$-diisopropylamino)phosphinyl acetates to generate the PACE Cap ODN. Automated synthesis for certain types of chimeras that require two oxidants is therefore limited by the programming capabilities of the ABI 394 instrument. For preparing ODNs such as PACE Cap, this reprogramming problem is minor, as it must be done only twice.

\section{DEPROTECTION, PURIFICATION, AND CHARACTERIZATION OF MODIFIED DNA}

The following steps are used for PACE, S-PACE, and chimeric DNA. After partial deprotection and cleavage from the solid support, the ODNs are easily purified by reversedphase HPLC (RP-HPLC) using the 5'-DMTr protecting group for hydrophobic affinity (trityl-on purification). After detritylation and further RP-HPLC purification, they are analyzed by ion-exchange HPLC to determine yield and by matrix-assisted laser desorption/ionization time-of-flight mass spectometry (MALDI-TOF-MS) to obtain ODN mass information.

\section{Materials}

DNA synthesis column containing oligodeoxyribonucleotides (ODN) linked to controlled-pore glass (CPG)

Acetonitrile, anhydrous

Argon or nitrogen, anhydrous

$1.5 \%(\mathrm{v} / \mathrm{v})$ 1,8-diazabicyclo-[5.4.0]undec-7-ene (DBU; Aldrich) in anhydrous acetonitrile

40\% methylamine, aqueous (Aldrich) 
RP-HPLC mobile phases:

A: $100 \mathrm{mM}$ triethylammonium acetate, $\mathrm{pH} 8.0$

$\mathrm{B}$ : acetonitrile

$10 \mathrm{mM}$ Tris $\cdot \mathrm{Cl}, \mathrm{pH} 8.0$ (APPENDIX 2A)

$80 \%$ acetic acid

$50 \mathrm{mM}$ triethylammonium acetate, $\mathrm{pH} 8$

TE buffer, $\mathrm{pH} 8.0$ (APPENDIX 2A)

IEX-HPLC mobile phases:

A: $10 \mathrm{mM} \mathrm{NaOH} / 80 \mathrm{mM} \mathrm{NaBr}$

B: $10 \mathrm{mM} \mathrm{NaOH} / 1.5 \mathrm{M} \mathrm{NaBr}$

2,4,6-Trihydroxyacetophenone monohydrate (THAP) matrix

Ammonium citrate

1:1 (v/v) acetonitrile/ $\mathrm{H}_{2} \mathrm{O}$

10-mL Luer-tip syringe

Male Luer-to-tubing connector (Aldrich)

Conical vial: 3-mL Reacti-Vial sealed with Teflon-bonded silicon Tuf-Bond discs

(Pierce), preferred

$55^{\circ} \mathrm{C}$ heating block (e.g., Reacti-Block Aluminum Heat-Block, Pierce) or oven

Speedvac evaporator (Savant)

RP-HPLC apparatus with 25-cm $\times$ 9.4-mm-i.d. Zorbax 300SB-C18 column

(Agilent Technologies)

Ion-exchange (IEX)-HPLC apparatus with 1-mL RESOURCE Q column

(Amersham Pharmacia Biotech)

100-well plate, gold plated

Voyager-DE STR Biospectrometry Workstation mass analyzer (PerSeptive

Biosystems)

\section{Recover CPG-bound ODN}

1. Remove the DNA synthesis column containing the ODN linked to CPG from the synthesizer. Wash with $10 \mathrm{~mL}$ anhydrous acetonitrile using a 10-mL Luer-tip syringe to push the solvent through the column.

2. Flush with a low-pressure stream of dry argon or nitrogen using a male Luer-totubing connector fitted onto the inert gas line and placing the Luer-tip snugly into the DNA synthesis column. Blow a mild, low-pressure stream through the column until the CPG flows freely as the column is rotated.

3. Open the column and pour the contents into a conical glass vial (preferably a 3-mL Reacti-Vial).

\section{Remove dimethylcyanoethyl group}

4. Place $1 \mathrm{~mL}$ freshly prepared $1.5 \%$ DBU solution into the vial containing CPG and tighten the cap containing the Teflon-bonded silicon disc onto the vial. Vortex to stir the contents and incubate $60 \mathrm{~min}$ at room temperature.

The vial can be vortexed occasionally during this deprotection reaction.

5. Vortex again and allow the CPG to settle into the conical portion of the vial. Remove the DBU solution using a clean Pasteur pipet and discard. Repeat three times using $1 \mathrm{~mL}$ acetonitrile each time to ensure complete removal of the DBU.

\section{Cleave ODN and remove exocyclic amine-protecting group}

Oligonucleotides with Phosphonoacetate and Thiophosphonoacetate Diesters

6. Add $1 \mathrm{~mL}$ aqueous $40 \%$ methylamine to the CPG and place the Teflon cap snugly on the vial. Heat the solution $15 \mathrm{~min}$ at $55^{\circ} \mathrm{C}$ by placing the vial in a preheated heating block or oven.

7. Remove the vial from the heat source, vortex, and place in an ice bucket.

\subsubsection{8}


8. Once cooled, remove the top of the vial and transfer the methylamine solution to a microcentrifuge tube using a glass Pasteur pipet. Be careful to allow the CPG to settle to the bottom of the glass vial before transferring the methylamine solution.

The conical shape of the Reacti-Vial aids this process and prevents transfer of CPG.

The residual CPG can be washed with an additional volume of cold methylamine solution to optimize recovery. Be careful, however, not to over-fill the microcentrifuge tube.

9. Evaporate solution to dryness, preferably in a centrifugal evaporator/concentrator (e.g., SpeedVac) without heating. Store dry mixture of oligodeoxyribonucleotide products in the sealed tube up to 6 months at $-20^{\circ} \mathrm{C}$.

\section{Purify ODN by RP-HPLC and remove dimethoxytrityl group}

10. Dissolve the crude oligodeoxyribonucleotide mixture in $0.5 \mathrm{~mL}$ water. Perform tritylon purification by RP-HPLC on a $25-\mathrm{cm}$ Zorbax 300SB-C18 column. Elute with RP-HPLC mobile phases using the following gradient at a flow rate of $1.2 \mathrm{~mL} / \mathrm{min}$ :

$\begin{array}{ll}\text { Duration } & \text { Mobile phase B } \\ 0-2 \mathrm{~min} & 8 \% \\ 2-27 \mathrm{~min} & 8 \% \text { to } 20 \% \\ 27-52 \mathrm{~min} & 20 \% \text { to } 80 \% .\end{array}$

Collect fractions containing the full-length product.

11. Concentrate under vacuum (e.g., SpeedVac) in a microcentrifuge tube and redissolve in $100 \mu \mathrm{L}$ of $10 \mathrm{mM}$ Tris. $\mathrm{Cl}, \mathrm{pH} 8$.

It is important to use a buffered solution at $\mathrm{pH} 7$ to 8 for dissolving these ODNs. At acidic pH, PACE and S-PACE ODNs are less soluble and can stick to the tube, which reduces recovery.

12. Remove the trityl group by treatment for $1 \mathrm{hr}$ with $1 \mathrm{~mL}$ of $80 \%$ acetic acid.

13. Concentrate to dryness (e.g., in a SpeedVac) and dissolve in $0.5 \mathrm{~mL}$ of $50 \mathrm{mM}$ triethylammonium acetate, $\mathrm{pH} 8$.

14. Repeat preparative HPLC with the $25-\mathrm{cm} \times 9.4-\mathrm{mm}$-i.d. Zorbax 300SB-C18 column to isolate the fully deprotected ODNs. Use the same gradient conditions as for tritylon isolation of ODNs (step 10). Concentrate the resulting products under vacuum (e.g., Speedvac) and store up to 6 months at $-20^{\circ} \mathrm{C}$ in $\sim 100 \mu \mathrm{l}$ TE buffer, $\mathrm{pH}$ 8.0.

\section{Characterize ODNs by IEX-HPLC}

15. To determine synthetic yield of full-length product, analyze by ion-exchange HPLC using a 1-mL RESOURCE Q column. Elute with IEX-HPLC mobile phases using a gradient of $0 \%$ to $100 \%$ IEX-HPLC mobile phase B over $45 \mathrm{~min}$ at a flow rate of $1.5 \mathrm{~mL} / \mathrm{min}$.

\section{Analyze ODNs by mass spectrometry}

16. Dissolve the modified ODN in water at a concentration of $100 \mu \mathrm{M}$.

17. Add $45 \mathrm{mg}(0.2 \mathrm{mmol})$ THAP matrix and $2 \mathrm{mg}(8.2 \mu \mathrm{mol})$ ammonium citrate to $500 \mu \mathrm{L}$ of $1: 1(\mathrm{v} / \mathrm{v})$ acetonitrile/water to form a supersaturated solution (a cloudy suspension). Allow suspended particles to settle to the bottom of the tube, forming a clear supernatant.

18. Pipet $1 \mu \mathrm{L}$ THAP matrix supernatant onto a gold-plated 100 -well plate. Pipet $1 \mu \mathrm{L}$ ODN solution onto the same location. Allow spot to dry.

There is no need for a desalting step because the ODN was purified by RP-HPLC.

Synthesis of Modified Oligonucleotides and Conjugates

4.24.19 
19. Insert the plate into a Voyager Biospectrometry Workstation mass analyzer. Perform all MALDI-TOF-MS measurements in the positive ion mode.

Table 4.24 .5 lists the mass spectrometry results from a series of phosphonoacetate, thiophosphonoacetate, and chimeric oligomers.

\section{COMMENTARY}

\section{Background Information}

For many years now, oligodeoxyribonucleotides (ODNs) and their analogs have proven to be indispensable for most research in biology and biochemistry. They are used to study various biological processes through sequencing, PCR applications, and directed mutagenesis, as well as to diagnose diseases and study gene expression (DNA chips), identify single nucleotide polymorphisms, and modulate or control gene expression. The latter application has broad implications from basic research on gene function to the use of ODNs as therapeutic agents. As one would expect, success in these areas has been possible because of extensive research using a large number of ODNs. As each analog investigated thus far has a specific repertoire of advantages and disadvantages, it is worthwhile to continue developing new derivatives useful for biological research. This objective led to the PACE and SPACE oligomers whose synthesis chemistries are outlined in this manuscript.

\section{Synthesis using phosphonoacetic acid}

Phosphonoacetic acid has been known as an effective mimic of phosphoric acid and its derivatives for many decades. At neutral to basic $\mathrm{pH}$, the three-dimensional structure and charge density of phosphonoacetate is remarkably similar to phosphate. The ability to mimic phosphate was demonstrated by the use of phosphonoacetic acid as an antiviral agent (Overby et al., 1977). Most methods for the synthesis of phosphonoacetic acid proceed through an oxidative transformation like an Arbuzov reaction (Arbuzov and Dunin, 1927), resulting in a phosphorus molecule in the oxidation state $\mathrm{P}(\mathrm{V})$. Once the carboxylic acid group is attached, the molecule becomes quite inactive to methods of coupling by activated transesterification. Even with these difficulties, phosphonoacetic acid was successfully coupled to a variety of nucleosides in an attempt to mimic $5^{\prime}$ - and $3^{\prime}$-phosphorylated nucleosides; these molecules also demonstrated antiviral activity (Griengl et al., 1988; Lambert et al., 1989).

The authors initially attempted to develop a method for synthesizing PACE ODNs using methyl $5^{\prime}-O$-(4,4'-dimethoxytrityl)-2'- deoxythymidine- $3^{\prime}-O$-phosphonoacetate prepared by the same methods developed for making phosphonoacetic acid-derived mononucleosides. This synthon was activated with various arylsulfonyl chlorides or 1-(2mesitylenesulfonyl)-3-nitro-1,2,4-triazole and condensed with 2 -deoxythymidine linked to a controlled-pore glass support. Unfortunately, coupling yields for these reactions never exceeded 5\%, even after $24 \mathrm{hr}$ (similar results were obtained with the acetic acid ethyl ester). A further problem with this approach was then discovered, namely that the alkaline conditions previously described to remove either the methyl or ethyl ester resulted in partial to complete destruction of the phosphonate internucleotide linkage. Due to these problems, it was concluded that $P(V)$ coupling would never produce a useful method for PACE DNA synthesis.

Rudolph et al. (1996) subsequently reported the ability to synthesize a 13-mer chimera having alternating phosphate and phosphonoacetate internucleotide linkages. Using the identical techniques previously described for making phosphonoacetic acid-derived mononucleosides, they were able to prepare a solutionphase 2'-deoxythymidine phosphonoacetate dimer as the acetic acid methyl ester and convert it to the $3^{\prime}$-phosphoramidite. Joining these dimers using classical phosphoramidite chemistry then produced the reported 13-mer chimera having alternating phosphonoacetate and phosphodiester linkages. Because the suspected 13-mer products were only characterized by relative HPLC retention times, it is unclear whether the methyl phosphonoacetate ester was completely removed from this molecule by extended base hydrolysis; however, this noteworthy manuscript reaffirmed the confidence that the successful synthesis of PACE oligodeoxyribonucleotides would require the development of novel P(III) methods.

\section{Synthesis using phosphinylacetic acid}

Although the literature is somewhat sparse, phosphinylacetic acids have been known for quite some time (Matrosov et al., 1972). These molecules were most often evaluated as potential solvents for charged heavy metal ions and used for applications such as the 
purification of uranium by selective solvolysis. As a result, these types of compounds have been characterized for their chemical and physical properties, but have never been described as reactive synthons in organic chemistry or used to prepare analogs of peptides, oligosaccharides, oligodeoxyribonucleotides, or lipids. Based upon the literature and the authors' experience, a potentially important reason for this lack of interest was the difficult challenge inherent in synthesizing these derivatives and the inaccurate characterization data based upon the early literature. A successful new approach was therefore developed as outlined in Figures 4.24.2 and 4.24.3. Initially, methyl 2'-deoxythymidine-3'-O- $(N, N$ diisopropylamino)phosphinyl acetate was synthesized and used to prepare a dimer having a PACE internucleotide linkage. The high isolated yield of this dimer $(81 \%)$ was extremely encouraging. Further studies, however, confirmed that removal of the methyl ester with various alkaline solutions led to degradation of the internucleotide linkage. As a consequence, the acetic acid 2-cyanoethyl ester was next investigated, as there was an expectation that it could be removed using a non-nucleophilic base such as DBU. Although this type of ester protection proved promising, it was not entirely satisfactory, as $\sim 8 \%$ of the PACE internucleotide linkages were further modified to phosphate under DBU treatment. These observations led the group to the sterically hindered acetic acid 1,1-dimethyl-2-cyanoethyl ester, which could be removed essentially quantitatively ( $<1 \%$ hydrolysis) with non-nucleophilic base.

\section{Removal of protecting groups}

A related early concern during the development of this chemistry was potential problems associated with removal of the amine-protecting groups commonly used on cytosine, adenine, and guanine. These blocking groups are routinely eliminated with mild base at elevated temperatures, conditions that are known to degrade alkyl phosphonate internucleotide linkages. An alternative was found in the method developed by Reddy et al. (1994), which uses $40 \%$ methylamine in water to rapidly remove exocyclic amine from ODNs containing $N$-acetyl- 2 -deoxycytidine residues without methylation of the exocyclic amine groups. By slightly modifying these conditions, it was possible to achieve complete removal of the heterobase-protecting groups in $15 \mathrm{~min}$ at $55^{\circ} \mathrm{C}$. The results showed that, under these conditions, both PACE and SPACE ODNs were relatively stable, as $1.4 \%$ and $1.2 \%$, respectively, of the internucleotide linkages were degraded in $15 \mathrm{~min}$. Mass spectroscopy of the resulting ODN products also demonstrated no methylation of cytidine residues protected by acetyl groups. Although not entirely satisfactory (the problem is under further investigation), this procedure is currently used in the authors' laboratory to remove the base-protecting groups.

\section{Compound Characterization}

Both ${ }^{1} \mathrm{H}$ and ${ }^{31} \mathrm{P}$ NMR are indispensable for analyzing precursors and synthons used to prepare these ODNs. ${ }^{1} \mathrm{H}$ NMR spectra are recorded on Varian VXR-300 and Bruker $400 \mathrm{MHz}$ spectrometers with tetramethylsilane as an internal standard. A Bruker $400 \mathrm{MHz}$ spectrophotometer and an external $85 \% \mathrm{H}_{3} \mathrm{PO}_{4}$ capillary are used for recording oligomer ${ }^{31} \mathrm{P} \mathrm{NMR}$ in $\mathrm{D}_{2} \mathrm{O}$. In the case of chimeras, the number of PACE, S-PACE, phosphate, and thiophosphate internucleotide linkages can be determined, as they have different ${ }^{31} \mathrm{P}$ NMR spectra. MALDI-TOF mass spectrometry (UNIT 10.1) should be used to analyze the molecular weight of these oligomers and to confirm that the correct base composition has been achieved. This is important because PACE and S-PACE ODNs are resistant toward degradation by nucleases. As a consequence, classical enzymatic methods cannot be used to characterize these ODNs. Further analysis of these ODNs is possible using standard 20\% polyacrylamide gel electrophoresis (UNIT 10.4) followed by visualization and densitometric scanning. It is also possible to derivatize these oligomers with fluorescein and use this label to visualize ODNs on polyacrylamide gels (Dellinger et al., 2003). In all cases, PACE and S-PACE ODNs exhibit electrophoretic mobilities similar to natural DNA of the same length.

\section{Critical Parameters and Troubleshooting}

\section{Reagent quality}

All nucleic acid chemists know that water (preferably the lack thereof) is the most critical parameter for synthesizing DNA, RNA, and their analogs. Certainly this rule applies to the synthesis of PACE and S-PACE ODNs. Among the various sources of water contamination, the most significant is acetonitrile, which must be anhydrous for several key synthesis steps. These include its use as a solvent for the synthons and tetrazole. Acetonitrile
Synthesis of Modified Oligonucleotides and Conjugates

4.24.21 
used to wash the CPG just prior to each condensation step should also be anhydrous. Currently, commercial synthesis-grade acetonitrile is usually sufficiently anhydrous for these steps. It should, however, be checked with a Karl Fisher titrator before use. In order to be absolutely sure that the acetonitrile is anhydrous, distillation from calcium hydride immediately before use is recommended. Another source of water is its adsorption on the synthons during storage. This can be minimized by storing the synthons at $-20^{\circ} \mathrm{C}$ under dry argon in a desiccator equipped with Drierite. Prior to opening the desiccator, it should be allowed to attain room temperature. Following transfer of the synthons to vials for use on the synthesizer, the samples should be dried under vacuum overnight to remove final traces of water.

In addition to water, there are other precautions that should be followed in order to ensure high repetitive yields during ODN synthesis. One is the quality of tetrazole used to activate the synthons. Commercially available material, if prepared specifically for oligodeoxyribonucleotide synthesis $(0.45 \mathrm{M}$ acetonitrile solution), is usually sufficiently pure for direct use; however, even reagent-grade tetrazole as obtained from various suppliers should be purified by sublimation before use in ODN synthesis. Another potential problem relates to the preparation of various synthons. During their synthesis, diisopropylammonium tetrazolide is generated. This salt must be completely removed during flash chromatography; otherwise, it will depress the overall ODN synthesis rate and yield by buffering the condensation reaction. Excessive amounts of this salt can be detected in the ${ }^{1} \mathrm{H}$ NMR spectra of the purified synthons.

\section{$P(I I I)$ to $P(V)$ oxidation}

Another potential problem that can be encountered during ODN synthesis is incomplete sulfurization or oxidation of $\mathrm{P}(\mathrm{III})$ to $\mathrm{P}(\mathrm{V})$. This leads to degradation of the oligomer, because trivalent phosphorus derivatives are known to be unstable to acid (a key step in the synthesis cycle is acid detritylation). It is difficult to monitor this problem during synthesis because the consequences (loss of stepwise trityl yields and overall deterioration of oligomer quality as observed by HPLC of the products) are common to several unrelated problems in DNA synthesis. If lack of complete oxidation is suspected, this step can be checked by ${ }^{31} \mathrm{P}$ gel-phase NMR (Greef et al., 1996); however, a more com- mon solution is to simply replace the oxidation and sulfurization reagents with fresh samples. Once again, it is important to use the correct concentration of the oxidizing agent. The phosphonite internucleotide linkage produced after the phosphonamidite coupling reaction is more easily oxidized than the phosphite triester internucleotide linkage produced after a standard phosphoramidite coupling reaction. The phosphonite internucleotide linkage can be quantitatively oxidized by a 3-min oxidation using 0.1 M CSO in anhydrous acetonitrile. However, the phosphite triester internucleotide linkage requires $0.5 \mathrm{M} \mathrm{CSO}$ for at least $3 \mathrm{~min}$. This is an important note for the synthesis of chimeric DNA sequences.

\section{Degradation}

It is well known that alkyl phosphonate internucleotide linkages are unstable toward basic conditions (Hogrefe et al., 1993). Phosphonoacetate and thiophosphonoacetate ODNs suffer from the same problem. For example, with aqueous $40 \%$ methylamine at $55^{\circ} \mathrm{C}$, $1.4 \%$ and $1.2 \%$, respectively, of the internucleotide linkages in these oligomers are degraded after $15 \mathrm{~min}$. It is therefore important to limit the deprotection time with this reagent to the minimum required for effective removal of the exocyclic amine-protecting group on cytosine, guanine, and adenine bases. A related potential problem is the use of DBU to remove the dimethylcyanoethyl protecting group. These conditions must be anhydrous. It is recommended that the DBU be dried over 4- $\AA$ molecular sieves prior to use and careful attention be given to the concentration of DBU used for removal of the dimethylcyanoethyl protecting groups. The presence of water in the DBU solution will generate hydroxide anions, resulting in degradation of PACE and S-PACE internucleotide linkages, and can also lead to the premature cleavage of the ODN from the solid support. It is important that the concentration of DBU $(1.5 \%)$ be accurately measured and that exposure of the CPG to this solution be limited to $60 \mathrm{~min}$. Significantly higher concentrations of DBU (even in the absence of water) or very long exposure times can result in premature cleavage of the ODN from the $\mathrm{CPG}$, reducing the recovery yields. Due to the deleterious effect of hydroxide ion on PACE and S-PACE internucleotide linkages, any base should be excluded from the chromatography solvents used to purify these synthons. As a consequence, chromatography must be carried out rapidly in order to minimize any detritylation, which can occur due to the acidity of the 
silica gel. The acidity of silica gel can vary greatly from batch to batch and from manufacturer to manufacturer. In the authors' experience, most batches of silica gel are not acidic enough to give any significant amount of detritylation. Furthermore, there is a considerable amount of diisopropylammonium tetrazolide in the crude $2^{\prime}$-deoxynucleoside- $3^{\prime}$ $O$-( $N, N$-diisopropylamino)phosphinyl acetate products to help neutralize some excess acid on the silica gel. Nonetheless, detritylation does occur, albeit infrequently, during column chromatography. Detritylation on the column is easily detectable as a bright orange band on the silica column. To eliminate this, a small amount of TEA may be used to neutralize excess acid on silica gel during column packing and equilibration, but excess amine should be thoroughly flushed from the column prior to adding the crude material to the top of the column.

\section{Deprotection of internucleotide linkages}

The use of anhydrous DBU deprotection of cyanoethyl protecting groups on ODNs can result in the undesired side product of N3 alkylation of thymidine residues from the production of acrylonitrile under these conditions. These products can easily be detected by mass spectroscopy (both MALDI-MS and LC/MS). This is one of the reasons that the use of the dimethylcyanoethyl protecting group is recommended over the more typical cyanoethyl protecting group. When protecting groups for the PACE and S-PACE internucleotide linkages were first evaluated, it was discovered that removal of the cyanoethyl protecting group using anhydrous DBU resulted in small amounts of these N3 alkylation products. By switching from cyanoethyl to dimethylcyanoethyl protection of the PACE and S-PACE internucleotide linkages, it was found that detectable amounts of N3 alkylation of the thymidine residues on the ODN products were eliminated, even as evaluated by highly sensitive LC/MS/MS techniques.

\section{Handling modified ODNs}

Once the PACE oligodeoxyribonucleotides are produced, it is important to realize that the internucleotide bond is a weaker acid $\left(\mathrm{p} K_{\mathrm{a}} \cong 3.8\right)$ than the phosphodiester bond of native DNA ( $\left.\mathrm{p} K_{\mathrm{a}} \cong 1.8\right)$. The implications in handling these modified oligodeoxyribonucleotides have to do with their solubility in water under acidic conditions. Phosphonoacetate internucleotide bonds can be protonated with weak acids such as acetic acid. Once proto- nated, the oligodeoxyribonucleotide is significantly less soluble in aqueous solutions. It is important that working solutions are buffered at neutral to slightly higher $\mathrm{pH}$ in order to prevent loss by precipitation and adherence to surfaces. This is especially important during trityl-on purification of the oligodeoxyribonucleotide. The final step is removal of the DMTr group using $80 \%$ glacial acetic acid followed by desalting on a reversed-phase column. The acetic acid is removed in a vacuum evaporator, leaving behind the modified ODN in its protonated form. In order to completely dissolve the ODN, it is important to use a buffer with enough capacity to neutralize the acidic sites on the backbone. This is accomplished by vortexing the sample vigorously during the initial resuspension of the ODN in a $\mathrm{pH}$ 8.0 triethylammonium acetate (TEAA) buffer, evaporating to dryness, and then redissolving the sample once again in TEAA prior to desalting.

\section{Anticipated Results}

1,1-Dimethyl-2-cyanoethyl $\quad 5^{\prime}-O-\left(4,4^{\prime}-\right.$ dimethoxytrityl)-2'-deoxynucleoside- $3^{\prime}-O$ ( $N, N$-diisopropylamino)phosphinyl acetates have been used to prepare ODNs up to $25 \mathrm{nt}$ in length. These oligomers contain PACE, S-PACE, phosphorothioate, and phosphate internucleotide linkages in various combinations and have isolated yields of $\sim 30 \%$. Several examples of those that have been synthesized, including their mass spectral analysis, are included in Table 4.24.5.

A typical HPLC profile as obtained by reversed-phase column chromatography (trityl-on) is shown in Figure 4.24.6. Although the peaks are broad, as expected due to the large number of diastereomers, it is clear that the expected product dominates the HPLC profile of the crude reaction mixture. When this oligomer is repurified by column chromatography after removal of the 4,4'dimethoxytrityl group, very few additional side products appear. At this stage of purification, these oligomers are essentially homogeneous (confirmed by analytical ion-exchange HPLC; Dellinger et al., 2003) and can be used for various biochemical experiments. A typical mass spectrum for the PACE Cap ODN (Table 4.24.5) is shown in Figure 4.24.7.

Oligomers with stable acetic acid esters (methyl and butyl) have also been prepared and tested for uptake by HeLa and SKBR3 cells. The results (Sheehan et al., 2003) show that these ODNs reversibly accumulate in the cytoplasm of cells (presumably
Synthesis of Modified Oligonucleotides and Conjugates

4.24.23 


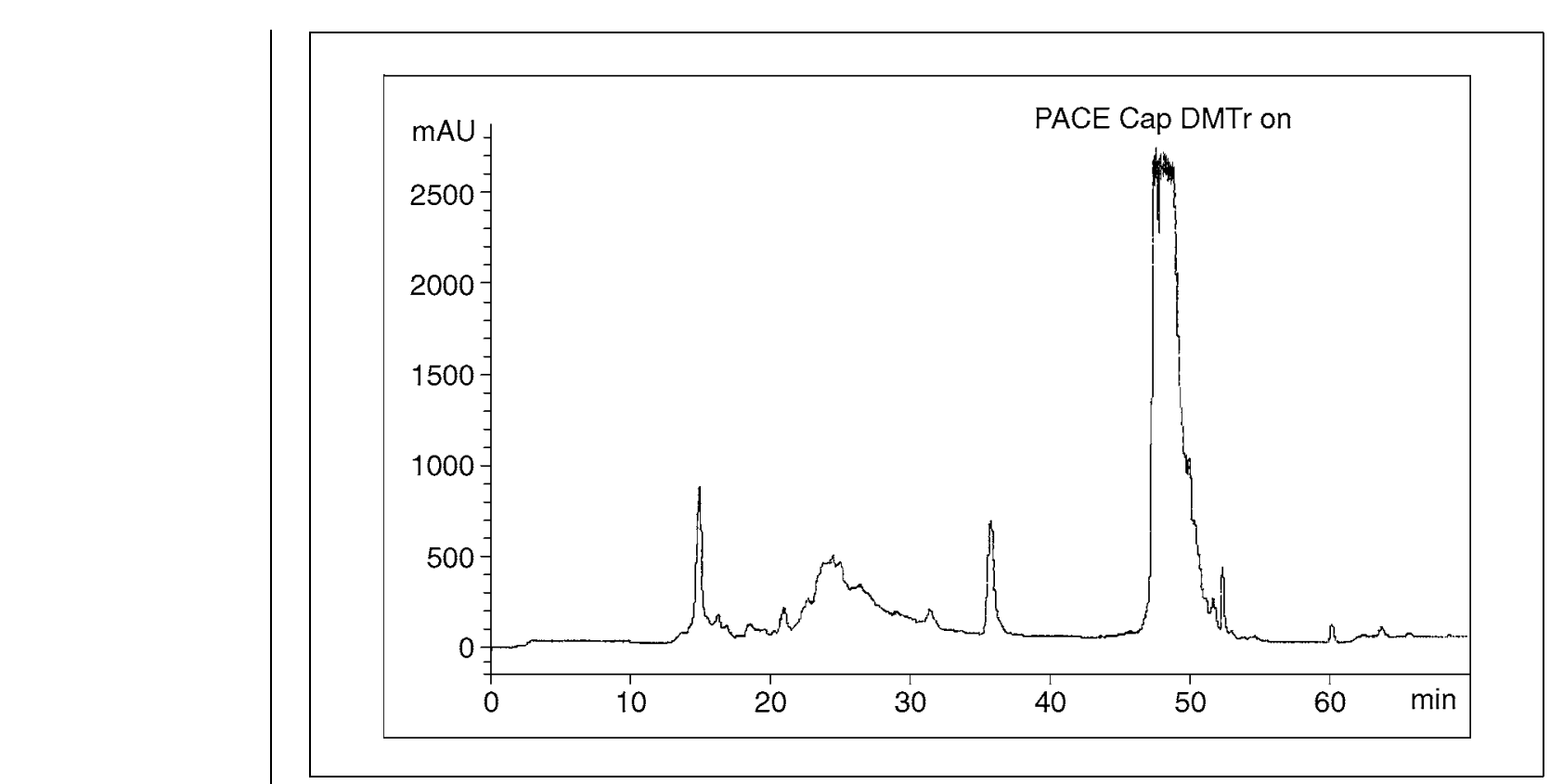

Figure 4.24.6 Reversed-phase column chromatography (trityl on) of the total reaction mixture obtained during the preparation of the PACE Cap ODN (see Table 4.24.5 for sequence). Gradient conditions and the column are described in the text.

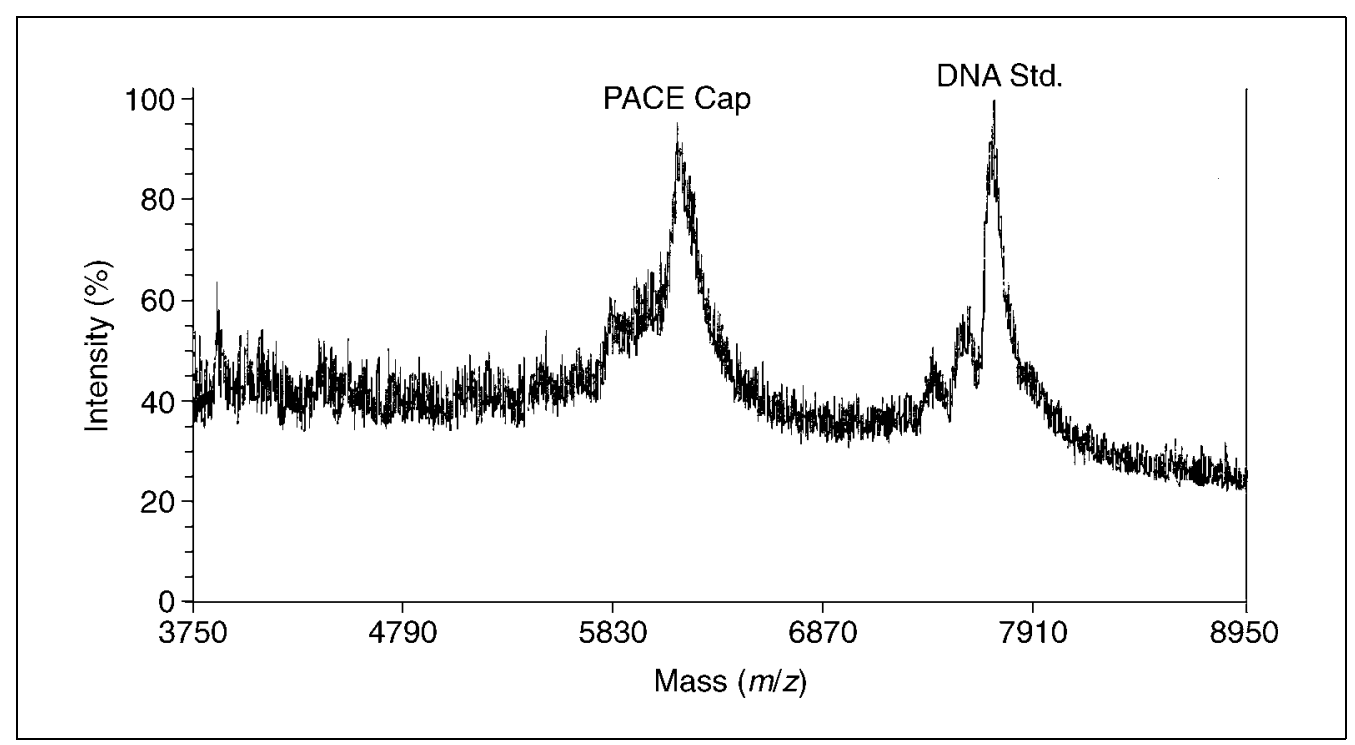

Figure 4.24.7 MALDI-TOF-MS analysis of PACE Cap ODN. The mass spectrum was determined using conditions described in the text.

by pinocytosis followed by ester hydrolysis). Current research in this area is focused upon enhancing in vivo acetic acid ester hydrolysis by using biologically relevant esters and studying these oligomers for antisense activity.

The isolated yields of PACE and S-PACE ODNs depend upon the length, sequence, and number of analog internucleotide linkages. This is because both PACE and S-PACE internucleotide linkages are somewhat labile toward aqueous $40 \%$ methylamine. These yields for 18-mers, based upon the amount of 2'-deoxynucleoside attached to $\mathrm{CPG}$, range from $20 \%$ to $30 \%$ following two reversedphase HPLC column purification steps (tritylon followed by trityl-off). The resulting oligomers are at least $95 \%$ homogeneous when evaluated by ion-exchange analytical column chromatography and are ready to be used for various biochemical experiments.

\section{Time Considerations}

Synthesis of 1,1-dimethyl-2-cyanoethyl [bis( $N, N$-diisopropylamino)phosphinyl] acetate can take up to four days. The procedure begins

\subsubsection{4}


with the preparation of 1,1-dimethyl-2cyanoethyl bromoacetate, which requires an overnight reflux of bromoacetyl bromide with 3-hydroxy-3-methylbutyronitrile in toluene. The next morning the reflux is stopped and the reaction mixture evaporated to an oil. The 1,1-dimethyl-2-cyanoethyl bromoacetate is purified by distillation, which requires 3 to $4 \mathrm{hr}$, and the day ends with ${ }^{1} \mathrm{H}$ NMR analysis of the bromoacetate distillate and ${ }^{31} \mathrm{P}$ NMR examination of the $\operatorname{bis}(N, N-$ diisopropylamino)chlorophosphine. Clean up of the reflux equipment and set up of the distillation apparatus can usually be accomplished during the evaporation process. The Reformatsky coupling reaction to yield 1,1-dimethyl-2-cyanoethyl [bis $(N, N$ diisopropylamino)phosphinyl]acetate will require 3 to $5 \mathrm{hr}$ to set up and complete. Initial isolation of the product by trituration and liquid/liquid extraction will take an additional 3 to $4 \mathrm{hr}$, including combining the hexane fractions and evaporating the hexane solutions to an oil. The product is then redissolved in hexanes and allowed to cool overnight to precipitate additional impurities. This solution is decanted, concentrated to an oil using a rotary evaporator, and characterized by NMR and mass spectroscopy. This final process can take at least 3 to $4 \mathrm{hr}$. If optional crystallization of the product from pentane is carried out, the total procedure will be extended by several hours to one day.

Preparation of the protected $2^{\prime}$ deoxynucleoside-3'-O-(N,N-diisopropylamino)phosphinyl acetates takes $\sim 2$ days. Phosphinylation of the protected 2 -deoxynucleoside is generally completed overnight, and the reaction mixture is evaluated the next morning by TLC and ${ }^{31} \mathrm{P}$ NMR. If the reaction is complete, triethylamine is added to quench the reaction and the product is purified by medium-pressure silica-gel column chromatography. This purification and isolation procedure usually requires 3 to $4 \mathrm{hr}$. For ease of handling, the product should be converted to a powder by precipitation into hexanes. This process usually requires only $30 \mathrm{~min}$, but the powder must be dried in a vacuum desiccator overnight in order to remove residual hexanes.

Set up for DNA synthesis requires attachment of various reagents and solvents to the DNA synthesizer. These include dichloromethane, acetonitrile, tetrazole (0.45 $\mathrm{M}$ in acetonitrile) the detritylation mixture, the two-part capping solution, and both oxidation and sulfurization reagents. This process requires $\sim 1 \mathrm{hr}$. The four appropriately protected 2'-deoxynucleoside-3'-O- $(N, N$ diisopropylamino)phosphinyl acetates are dissolved in anhydrous acetonitrile and the bottles placed on the DNA synthesizer. The DNA synthesis cycle for adding one 2 -deoxynucleotide requires $\sim 45 \mathrm{~min}$. Thus, the automated synthesis of a 20 -mer requires $\sim 15 \mathrm{hr}$.

Deprotection and cleavage of the ODN from CPG takes $\sim 2 \mathrm{hr}$. Major steps are removal of the dimethylcyanoethyl group with anhydrous DBU (60 min) and treatment with aqueous methylamine $\left(15 \mathrm{~min}, 55^{\circ} \mathrm{C}\right)$, which cleaves the oligomer from $\mathrm{CPG}$ and removes exocyclic amine-protecting groups. Following these steps, the reaction mixture is cooled and transferred to a microcentrifuge tube for concentration to dryness ( 1 to $2 \mathrm{hr}$ ).

The ODNs are purified by reversed-phase HPLC using the 4,4'-dimethoxytrityl group for hydrophobic affinity. The entire procedure requires $\sim 12 \mathrm{hr}$. The first step is trityl-on RPHPLC (50 min) followed by collection of appropriate product fractions and concentration to dryness (2 to $3 \mathrm{hr}$ ). Next, the $4,4^{\prime}$ dimethoxytrityl group is removed with aqueous $80 \%$ glacial acetic acid $(90 \mathrm{~min}$, room temperature) followed by concentration to dryness in a centrifugal concentrator. The ODN is then repurified and desalted by RP-HPLC. The same column and gradient can be used for both purifications. Appropriate product fractions are collected, combined, and concentrated to dryness. The resulting products are usually stored up to 6 months at $-20^{\circ} \mathrm{C}$ in $\mathrm{TE}$ buffer, pH 8.0.

\section{Literature Cited}

Arbuzov, A.E. and Dunin, A.A. 1927. Über phosphon-carbonsäuren. Ber. 60B:291-295.

Bayless, P.L. and Hauser, C.R. 1954. A Reformatskii type condensation of aroyl chlorides with ethyl 2-bromoisobutyrate by means of zinc to form $\beta$-keto esters. J. Am. Chem. Soc. 76:2306-2308.

Caruthers, M.H., Barone, A.D., Beaucage, S.L., Dodds, D.R., Fisher, E.F., McBride, L.J., Matteucci, M., Stabinsky, Z., and J.-Y. Tang. 1987. Chemical synthesis of deoxyoligonucleotides by the phosphoramidite method. Methods Enzymol. 154:287-313.

Dellinger, D.J., Sheehan, D.M., Christensen, N.K., Lindberg, J.G., and Caruthers, M.H. 2003. Solid phase chemical synthesis of phosphonoacetate and thiophosphonoacetate oligodeoxyribonucleotides. J. Am. Chem. Soc. 125:940-950.

Glen Research. 1996. Non-aqueous oxidation with 10-camphorsulfonyl-oxziridine. Glen Research Corporation Technical Report 9:8-9.
Synthesis of Modified Oligonucleotides and Conjugates

4.24.25 
Greef, C.H., Seeberger, P.H., Caruthers, M.H., Beaton, G., and Bankaitis-Davis, D. 1996. Synthesis of phosphorodithioate RNA by the H-phosphonothioate method. Tetrahedron Lett. 37:4451-4454.

Griengl, H., Hayden, W., Penn, G., Declercq, E., and Rosenwirth, B. 1988. Phosphonoformate and phosphonoacetate derivatives of 5-substituted 2 -deoxyuridines - synthesis and antiviral activity. J. Med. Chem. 31:1831-1839.

Hogrefe, R.I., Reynolds, M.A., Vaghefi, M.M., Young, K.M., Riley, T.A., Klem, R.E., and Arnold, L.J. Jr. 1993. An improved method for the synthesis and deprotection of methylphosphonate oligonucleotides. In Protocols for Oligonucleotides and Analogs, Vol. 20 (S. Agrawal, ed.) pp. 143-164. Humana Press, Totowa, New Jersey.

Lambert, R.W., Martin, J.A., Thomas, G.J., Duncan, I.B., Hall, M.J., and Heimer, E.P. 1989. Synthesis and antiviral activity of phosphonoacetic and phosphonoformic acid-esters of 5-bromo2 -deoxyuridine and related pyrimidine nucleosides and acyclonucleosides. J. Med. Chem. 32:367-374.

Matrosov, E.I., Tsvetlsov, E.N., Malevannaya, R.A., and Kabachnik, M.I. 1972. Infrared spectra and association of phosphinylacetic acid. $Z h$. Obshch. Khim. 42:1695-1700.

Overby, L.R., Duff, R.G., and Mao, J.C. 1977. Antiviral potential of phosphonoacetic acid. Ann. N.Y. Acad. Sci. 284:310-320.

Reddy, M.P., Hanna, N.B., and Farooqui, F. 1994. Fast cleavage and deprotection of oligonucleotides. Tetrahedron Lett. 35:4311-4314.
Rudolph, M.J., Reitman, M.S., MacMillan, E.W., and Cook. A.F. 1996. Phosphonoacetate derivatives of oligodeoxyribonucleotides. Nucleosides Nucleotides 15:1725-1739.

Sheehan, D., Lunstad, B., Yamada, C.M., Stell, B., Caruthers, M.H., and Dellinger, D.J. 2003. Biochemical properties of phosphonoacetate and thiophosphonoacetate oligodeoxyribonucleotides. Nucl. Acids Res. 31:4109-4118.

\section{Key References}

Dellinger et al., 2003. See above.

This reference outlines the chemistry used to prepare phosphonoacetate and thiophosphonoacetate ODNs from protected 2'-deoxynucleoside-3'$O$-(N,N-diisopropylamino)phosphinyl acetate synthons.

Sheehan et al., 2003. See above.

This reference presents initial biochemical and biophysical results with phosphonoacetate and thiophosphonoacetate ODNs.

Contributed by Douglas J. Dellinger

Agilent Laboratories

Boulder, Colorado

Christina M. Yamada and

Marvin H. Caruthers

University of Colorado

Boulder, Colorado

\section{Oligonucleotides with Phosphono- acetate and Thiophosphono- acetate Diesters}

